\title{
Article \\ Barriers and Enablers for the Adoption of Sustainable Manufacturing by Manufacturing SMEs
}

\author{
Claudia Lood Alayón ${ }^{1}$, Kristina Säfsten ${ }^{1, *(\mathbb{D})}$ and Glenn Johansson ${ }^{2,3}$ (D)
}

1 Department of Industrial Product Development, Production and Design, School of Engineering Jönköping University, 55111 Jönköping, Sweden; claudia.alayon@ju.se

2 Department of Design Sciences, Lund University, 22100 Lund, Sweden; glenn.johansson@design.lth.se

3 School of Innovation Design and Engineering, Mälardalen University, 63105 Eskilstuna, Sweden

* Correspondence: kristina.safsten@ju.se

check for updates

Citation: Alayón, C.L.; Säfsten, K.; Johansson, G. Barriers and Enablers for the Adoption of Sustainable Manufacturing by Manufacturing SMEs. Sustainability 2022, 14, 2364. https://doi.org/10.3390/su14042364

Academic Editor: Sooksan Kantabutra

Received: 30 December 2021 Accepted: 15 February 2022 Published: 18 February 2022

Publisher's Note: MDPI stays neutral with regard to jurisdictional claims in published maps and institutional affiliations.

Copyright: (c) 2022 by the authors. Licensee MDPI, Basel, Switzerland. This article is an open access article distributed under the terms and conditions of the Creative Commons Attribution (CC BY) license (https:// creativecommons.org/licenses/by/ $4.0 /)$.

\begin{abstract}
Small- and medium-sized enterprises (SMEs) have inherent characteristics, which require specific solutions for improving the sustainability performance of their operations. The purpose of this paper is to increase the knowledge on barriers and enablers for the adoption of sustainable manufacturing by manufacturing SMEs and to provide insights into what enablers can be used to overcome existing barriers. Taking, as a starting point, a systematic literature review, this paper presents a categorization of barriers and enablers for the adoption of sustainable manufacturing by manufacturing SMEs. In total, seven categories for classifying the barriers and enablers for the adoption of sustainable manufacturing within SMEs were identified: organizational, managerial and attitudinal; informational; governmental; financial; training and skills development; market and business context; and technological. Additionally, this study elaborates on what barriers could be mitigated through the enablers. This study found specific enablers with the potential to mitigate a significantly higher number of barriers and referred to them as 'critical enablers'. SMEs aiming to adopt sustainable manufacturing practices or improve their sustainability performance are encouraged to focus on the enablers in these categories. This paper synthesizes and facilitates interpretation of the existing body of evidence on barriers and enablers for adopting sustainable manufacturing in SMEs.
\end{abstract}

Keywords: SMEs; sustainable manufacturing; enablers; barriers; systematic literature review; categorization

\section{Introduction}

Small- and medium-sized enterprises (SMEs) have received little attention in the ongoing global debate regarding sustainability developments [1]. While the contributions of large organizations to the global anthropogenic impact on the environment and their effect on sustainability are obvious, the pivotal role of SMEs also requires urgent recognition. The key role of SMEs in global sustainability is easier to appreciate when the impacts of SMEs are viewed collectively. Statistics indicate that more than $95 \%$ of enterprises in the world are SMEs, accounting for approximately $60 \%$ of private sector employment [2]. Although the environmental impact of an individual SME may be limited, the cumulative environmental impact of SMEs can be high due to their large number [3]. An often quoted estimate on the environmental impact of SMEs indicates that SMEs generate up to 70\% of all global pollution [4]. Within the European Union, SMEs are believed to be responsible for $64 \%$ of pollution [3]. In short, the large number of SMEs means that, collectively, they have a substantial collective impact on the environment [4-6].

Based on the definition of sustainable development [7], the concept of sustainable manufacturing emerged. De Ron [8] defined it as an industrial activity that generates products which meet the needs and wishes of the present society without sacrificing the ability of future societies to meet their needs and wishes. Previous studies e.g., [9,10] have cited or proposed definitions based on the definition developed by the U.S. Department of 
Commerce [11] that defines sustainable manufacturing as "the creation of manufactured products using processes that minimize negative environmental impacts, conserve energy and resources, are safe for employees, communities, and consumers and are economically sound". However, recent studies have revealed a lack of consensus among researchers on the core understanding of sustainable manufacturing, ranging from different interpretations of the "sustainability" concept, to specific terms used to define and to set the focus of domains for the implementation of sustainable manufacturing [12]. Based on the above, in this study, sustainable manufacturing is defined from a triple bottle line context that considers the three dimensions of sustainability (environmental, social, and economic) within the manufacturing function, or the manufacturing business element. We apply the definition of sustainable manufacturing practices as the actions, initiatives, and techniques that positively affect the environmental, social or economic performance of a manufacturing company, helping to control or mitigate the impacts of the manufacturing operations on the triple bottom line [13].

A logic starting point for studying how SMEs adopt sustainable manufacturing practices, and the means for supporting SMEs, is to increase the understanding of the barriers and enablers for adopting such practices within SMEs. Studying the enabling and hindering factors affecting exclusively SMEs is essential as these organizations have inherent characteristics and face unique, local, contextual barriers, which require specific solutions. It is essential to realize an SME is different from a large organization; SMEs cannot be regarded as "little big businesses" [14,15].

Earlier research on the barriers and enablers faced by SMEs have addressed barriers to innovation in SMEs [16,17], barriers to SMEs' performance [18], barriers to environmentallysustainable manufacturing practices [19], and enablers for the design of sustainable production processes, products, and services [20], among others. The literature on barriers and/or enablers for sustainable manufacturing in SMEs is scattered, and only a few studies exhibit a categorization of the factors e.g., [21-24]. However, most of those studies failed to: describe the barriers and enablers individually; explain the logic underpinning the categorization of the factors; and define the categories precisely. Unsurprisingly, this body of literature does not include a systematic review on the topic that simultaneously considers the triple bottom line approach. Recently, Bhanot et al. [10,25] addressed the barriers and enablers for adopting sustainable manufacturing, but their studies did not focus exclusively on manufacturing SMEs (including data from large organizations and SMEs). It is evident that there is a lack of insight in the literature regarding the particular barriers and enablers surrounding the adoption of sustainable manufacturing practices, considering the three dimensions of sustainability, and focusing exclusively on manufacturing SMEs. Given this, further research into these factors is of utmost importance.

Classification schemes and overviews can be very helpful in organizing information from multiple sources, in this particular study, to organize the scattered information on barriers and enablers encountered by manufacturing SMEs adopting sustainability practices. Existing overviews on barriers and enablers focus mainly on energy efficiency [26-28] and do not consider barriers and enablers from a triple bottom line perspective. Likewise, existing overviews do not consider organizational size when identifying the barriers and enablers and include data from both large companies and SMEs. It is, therefore, important to compile an overview that organizes the barriers and enablers relevant to manufacturing SMEs, while adopting sustainable manufacturing from a triple bottom line perspective.

With the aforementioned in mind, the purpose of this paper is to increase the knowledge on barriers and enablers for the adoption of sustainable manufacturing by manufacturing SMEs and thereby provide insights into how to overcome existing barriers to sustainability through better leverage of the enablers. To fulfill this purpose, two research questions were formulated:

RQ1: What are the barriers and enablers faced by manufacturing SMEs related to the adoption of sustainable manufacturing practices?

RQ2: What enablers might be used to mitigate the barriers? 
To answer these research questions, our study systematically reviewed the existing body of literature related to barriers and enablers for sustainable manufacturing within manufacturing SMEs, categorized the identified barriers and enablers, and, finally, pointed out enablers with the potential to address a several of the barriers (the "critical enablers").

Increased knowledge of the barriers and enablers affecting SMEs will, on the one hand, help SMEs to achieve basic sustainability objectives by enhancing their knowledge about how to address barriers to sustainability through better leverage of sustainability enablers [29]. On the other hand, a better understanding of the barriers and enablers for adopting sustainable manufacturing will help in the development of frameworks to encourage proactive environmental behaviors among SMEs and, thus, foster better sustainable manufacturing practices [30].

We believe that this study makes the following contributions: firstly, it helps to correct the paucity of research about barriers and enablers for sustainable manufacturing in manufacturing SMEs from a triple bottom line perspective. Secondly, it constitutes a rare effort to present a systematic review of the existing literature on enablers and barriers to the implementation of sustainable manufacturing in manufacturing SMEs. Thirdly, it raises awareness among academics and manufacturing SMEs stakeholders concerning the current barriers SMEs are facing, while adopting sustainable manufacturing. In addition, finally, it provides a foundation for future studies aiming to explore the perceptions of SMEs stakeholders (e.g., large company customers and government) on the barriers and enablers presented in this paper.

The paper is structured as follows. Section 2 introduces relevant definitions. Section 3 provides methodological details about how the systematic review was conducted. Section 4 presents the overview and categorization of identified barriers and enablers, as well as reports the review findings. Section 5 discusses the findings and limitations of the study, and Section 6 presents the conclusions.

\section{Theoretical Framework: Barriers, Enablers, and SMEs}

'Barriers' and 'enablers' are two key terms in this paper. Previous research has identified barriers and enablers for sustainable manufacturing e.g., [10], enablers for environmental manufacturing practices in both large enterprises and SMEs [31], barriers and enablers for sustainable supply chain management e.g., [32], and barriers and enablers for sustainable manufacturing in both large enterprises and SMEs within a specific country [33], and industrial barriers to sustainable manufacturing e.g., [34]. Furthermore, previous research has also established that these factors can be either internal or external to the organization e.g., [34-36]. Some examples of barriers to sustainable manufacturing are lack of knowledge concerning how to adopt sustainable manufacturing practices [37], too high costs [10], or resistance to change [21,38]. Enablers for sustainable manufacturing could, for example, be managerial leadership toward sustainability [39] or government promotion and regulations [10].

A barrier is defined as 'something that prevents or hinders movement or action' [40]. Synonyms of the term 'barrier' found in the literature include 'challenges', 'obstacles', and 'hindering factors'. Likewise, an 'enabler' is defined as 'something that makes it possible for a particular thing to happen or be done', with the term 'enabler' being associated with terms/phrases, such as 'helpers', 'facilitators', and 'having the competence or ability to attain a certain goal or to implement a process' [41]. Based on the above, in this paper, 'enablers' are defined as the external factors or internal attributes of SMEs that are likely to assist, facilitate, or contribute to the adoption of sustainable manufacturing practices. Similarly, 'barriers' are defined as external factors or internal attributes of SMEs that likely represent a current or future difficulty or obstacle for SMEs wishing to adopt sustainable manufacturing practices. The terms 'drivers' and 'enablers' are sometimes used interchangeably $[19,42]$, while others have stated that the definition of drivers itself is debatable [43]. 
Previous studies have also stressed the importance of indicating the internal or external nature of barriers and enablers [44]. In this study, we distinguish between 'internal' and 'external' barriers and enablers, depending on their origin (relative to an SME) and the ability an SME has to control them. Hence, internal barriers or enablers refer to factors that hinder or facilitate the adoption of sustainable manufacturing practices, and that originate within the organization and, thus, can be directly controlled by it. However, external barriers and enablers constitute the factors which hinder or facilitate the adoption of sustainable manufacturing practices that have an external origin with respect to the organization and, thus, cannot be directly controlled by it.

Another key term in this paper is 'SME'. Although a universal definition of what constitutes an SME does not exist, the most common definitions used by regulators are based on the number of employees, sales, and/or loan size [45]. The number of employees can vary across countries, where the most frequent upper limit designating an SME is 250 employees, as in the European Union [46]. For the purpose of this paper, we have considered the definition given by the European Commission [47], where an SME is an enterprise which employs a maximum of 250 employees and has a maximum turnover of $€ 50$ million, or a total balance sheet of $€ 43$ million. SMEs have also been characterized as heterogeneous organizations with particular qualities (pertaining to size, resources, management style, and relationships) that make it difficult for them to adopt the practices of large firms [15]. Commonly-mentioned characteristics of SMEs include: flat organizational structures, unclear roles with low degrees of specialization, short-term priorities, a focus on daily operations, lack of external orientation, tacit knowledge based mainly on experience, flexibility and responsiveness, entrepreneurial orientation, and limited resources [48,49]. SMEs have also been described as being able to respond to the needs and preferences of potential customers more quickly than larger organizations [50].

Actions in SMEs traditionally occur in response to operational needs, with the focus on daily operations and short-term solutions [51]. The prevailing learning strategy within SMEs, learning by doing, mainly supports technical skills and know-how, and it is less effective for transferring managerial knowledge and practices [52]. Hence, the focus in SMEs is on technical and manufacturing-related competencies, rather than on management practices, which may create barriers to enhanced sustainable manufacturing practices. It is worth noting that SMEs encounter difficulties when attempting to meet environmental and social standards, mainly due to insufficient financial and human resources and difficulties in accessing and managing information [42,53].

\section{Research Methodology}

This study follows a integrative view of sustainable manufacturing, by considering the triple bottom line approach [54]. Thus, it includes a larger and comprehensive set of barriers and enablers for sustainable manufacturing than earlier studies that have typically focused on barriers and/or enablers for the adoption of environmentally sustainable practices e.g., $[55,56]$.

Systematic literature reviews are recognized as "a standard methodology for locating, selecting, and appraising research and transferring the synthesized findings not only to researchers and academics but also to practitioners and policy-makers in a digestible format to inform action" [57] (p. 673).

Figure 1 depicts the process used to conduct the review, following a systematic process [57-59]. The systematic review process started with the development of a literature review protocol, which comprised the purpose of the review, the search strategy, search queries, inclusion and exclusion criteria, data extraction, and synthesis. 


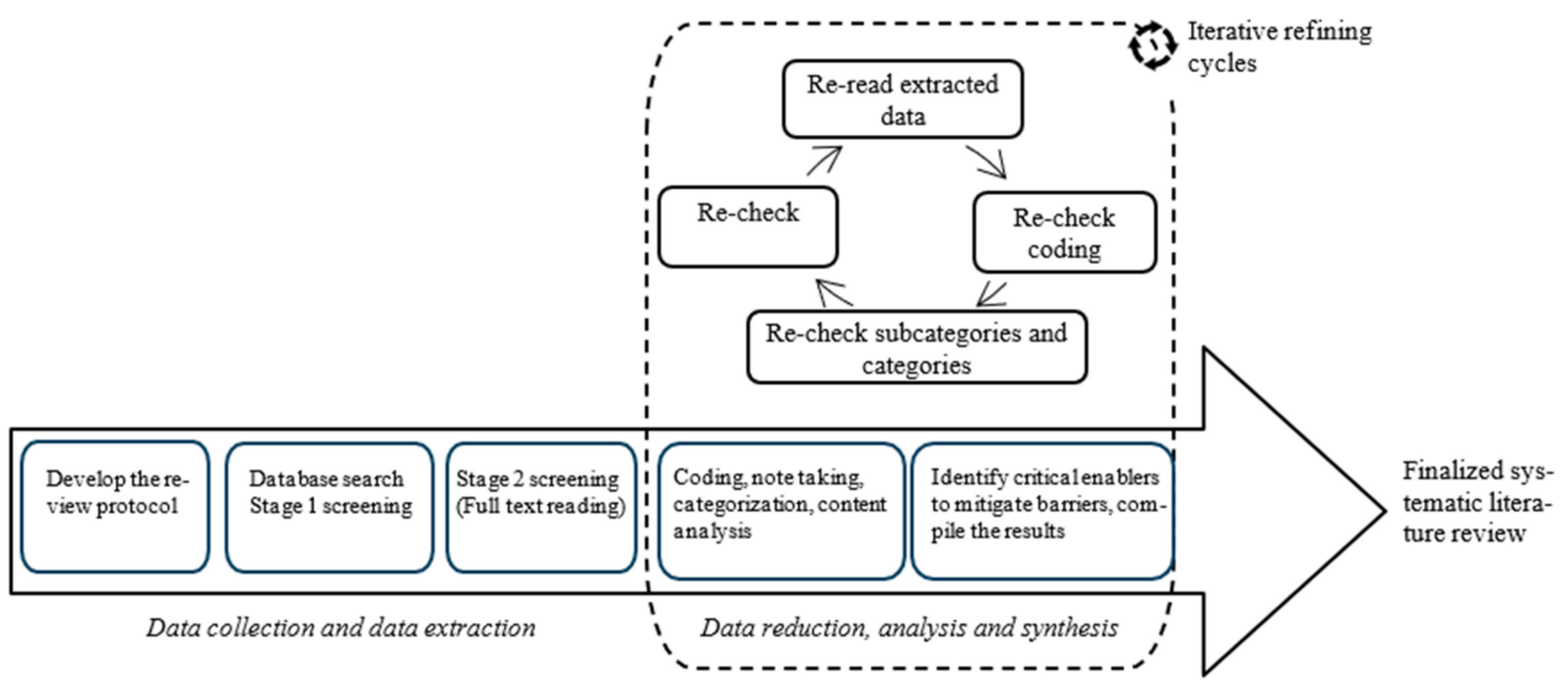

Figure 1. Systematic literature review process.

\subsection{Data Collection and Extraction}

Data collection comprised an electronic search of the literature, application of eligibility criteria to the search results, extraction of data from the final sample, and storage of data within a matrix in Microsoft Excel. The search strategy involved a limitation to journal papers to ensure high academic quality. The search was conducted in Scopus, the largest multidisciplinary bibliographic database of peer-reviewed literature. A structured keyword search query making use of basic Boolean operators and synonyms of relevant terms (see Table 1) ensured that all available papers addressing enablers and/or barriers for the adoption of sustainable manufacturing by SMEs were considered. The decision to search a large multidisciplinary bibliographic database instead of specific journals was made because journal papers that address sustainability concerns for manufacturing SMEs are published in journals associated with many different disciplines (e.g., operations management, environmental sciences, engineering, etc.).

Table 1. Search query used during the electronic search (the search was done during 2019).

\begin{tabular}{ccc}
\hline Search Terms & Where & Number of Hits \\
\hline (SME* OR "small and medium sized enterprise*" OR $^{*}$ & \\
"small compan*" OR "small enterprise*") AND & \\
(Sustainab* OR respons* OR environmental* OR & Scopus & \\
social* OR economic* OR green* OR clean* OR & (Title, abstract, & 2545 \\
benign) AND (enabl* OR barrier* OR challeng* OR & keywords) \\
"critical factor*" OR facilitator* OR driver* OR \\
hinder* OR obstacle*) AND (manufactur* OR \\
product* OR assemb* OR operation*)
\end{tabular}

The scope of the review was limited by the inclusion and exclusion criteria. Papers were included in the review if they: (i) concerned barriers and enablers for the adoption of sustainable manufacturing by SMEs; (ii) were published in a peer-reviewed journal between 1997 and 2018; (iii) were written in English; (iv) concerned SMEs within manufacturing industries that produced assembled products and had industrial processes; and (v) were related to operations management or industrial engineering. The scope of this review pertains to sustainable manufacturing; therefore, papers on aspects, such as eco-design, end-of-life product recovery, sustainability in product-service systems, eco-innovation, sustainable consumption, and sustainability in re-manufacturing, were excluded. 
Having located potentially relevant papers in Scopus, the two-stage screening process commenced, based on the inclusion and exclusion criteria above. The criteria played a significant role in discarding irrelevant papers and, therefore, in data reduction. The screening process was carried out in two stages. During the first stage, the inclusion and exclusion criteria were applied to the titles and abstracts of the initial sample of 2545 papers. The reasons for excluding papers at this stage were that the research did not relate to operations management or industrial engineering (740 papers), sustainability was not a major issue (583 papers), manufacturing was not addressed (416 papers), papers were of the conference type (350 papers), publications were books chapters (156 papers), papers were written in other languages than English (21 papers), and papers were duplicates (10 papers). After the first stage, the sample was reduced to 269 papers. The second stage comprised full-text reading of each paper remaining in the sample. During this stage, papers were excluded for the following reasons: did not address barriers and/or enablers for sustainable manufacturing adoption (199 papers); did not focus on SMEs context (24 papers); did not focus on sustainability (11 papers); did not apply to the European Commission's definition of SME (3 papers); or did not focus on manufacturing SMEs (1 paper). The second stage reduced the sample to 32 papers. When the final sample was identified, the next step was to extract relevant data from each paper. A Microsoft Excel Matrix was used to organize and summarize the data extracted from each paper for subsequent analysis. The matrix contained key aspects from each paper, such as: title, author, journal, year of publication, research method, sustainability perspective adopted by the paper, industry, small summary of the paper, and relevant findings.

\subsection{Data Reduction, Analysis, and Synthesis}

The analysis consisted of two phases: in the first phase, barriers and enablers were categorized; in the second phase, the enablers were matched with barriers in order to identify potential ways to mitigate the identified barriers. During the first phase, data was analyzed using content analysis, which involved the identification of codes, subcategories, and categories [60]. Codes served to reduce and analyze data. A code constitutes a "label that assign symbolic meaning to the descriptive or inferential information compiled during a study" [61] (p. 62). Parts of text that appear to capture key thoughts in each paper were highlighted to derive codes [60]. Codes resulted from inductive reasoning, that is, by defining the codes based on the raw data collected from the sample of papers (and not by defining codes and categories before the data collection took place).

Thereafter, conceptual or thematic relationships among the initial codes were sought after, merging similar codes to form subcategories (i.e., enablers and barriers). Furthermore, the subcategories were combined and grouped, based on the relationships between them, into a smaller number of categories [60]. Hence, this integration of several subcategories led to the emergence of the categories. An independent coder (a second author) revisited and discussed the coding and findings with the first author who carried out the coding, to agree or disagree with the categories and subcategories identified. It is worth noting that the process of reading the extracted data, creating codes, subcategories and categories, and re-checking these codes, categories and subcategories, and results was a cyclical process; see Figure 1. It is unlikely that these activities will be done once only $[58,61]$. Thus, the data reduction, analysis and synthesis activities were non-linear, instead of constituting an interactive and continuous process [61]. These iterative cycles are especially important for coding and refining the categories, as it complements or strengthens the inter-coder reliability of the review [62].

The second phase of the analysis aimed to identify enablers with the potential to mitigate the identified barriers. This phase began by grouping barriers and enablers belonging to the same category and identifying the enablers with the potential to mitigate a corresponding barrier. Then, each barrier was contrasted or matched up with the entire list of identified enablers (inter-category analysis) aiming to identify ways of mitigating a corresponding barrier. It is worth noting that, for matching up barriers with enablers, 
the descriptions or definitions of the barriers and enablers were of great help in making connections between the enablers that might mitigate a specific barrier. The outcome of the second phase of the analysis provides insights into how to address the identified barriers for adopting sustainable manufacturing in SMEs.

\section{Results}

\subsection{Descriptive Analysis of the Systematic Literature Review Sample}

The final sample contained 32 papers and included both conceptual papers and empirical papers presenting the barriers and enablers for adopting sustainable manufacturing in SMEs. Figure 2 shows the proportion of the publications in relation to their year of publication, the applied research methodology, and the sustainability dimension addressed.

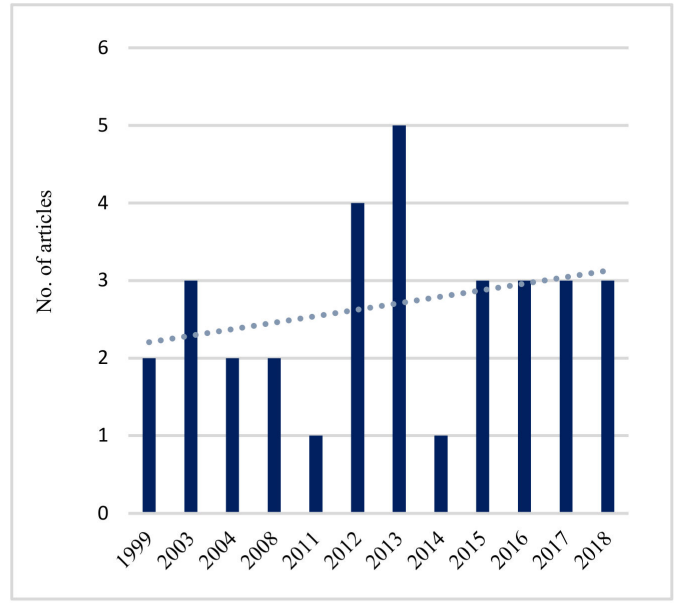

(a)

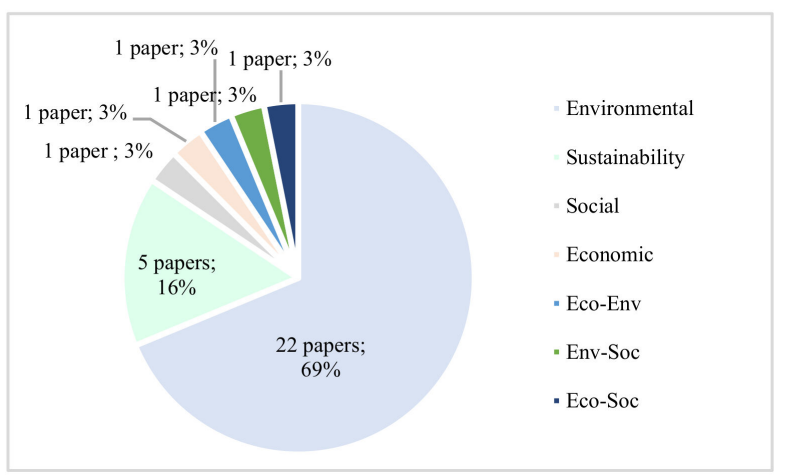

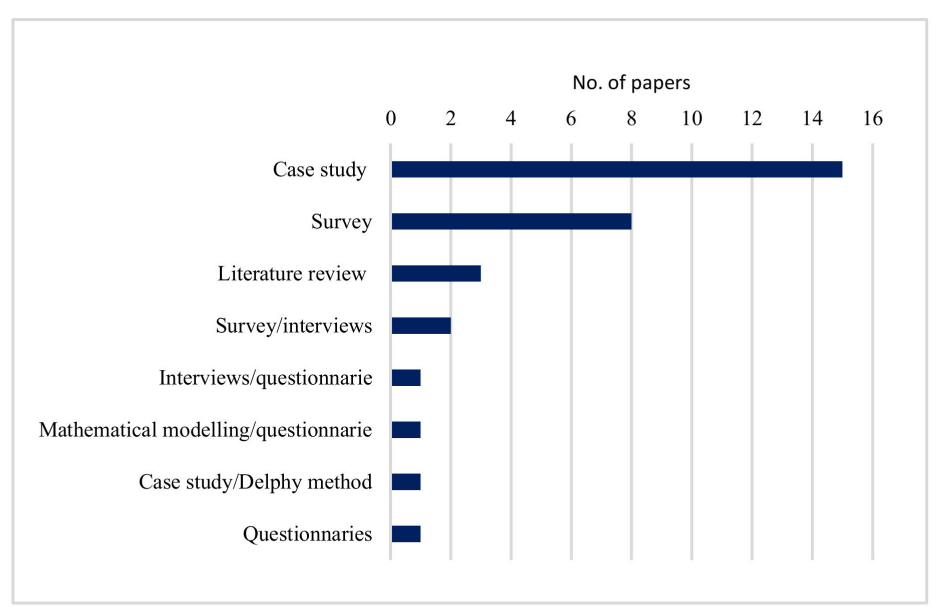

(b)

(c)

Figure 2. Descriptive data: (a) distribution of papers by year, (b) distribution of papers by research methodology), (c) distribution of papers by sustainability issue addressed.

The papers in the selected sample were quite evenly distributed between the years; see Figure 2a, with the exception of 2011 and 2014, with only one paper, respectively. This is, however, compensated with more papers 2012 and 2013, (four and five, respectively). Case studies ( $47 \%$ of papers) and surveys ( $25 \%$ of papers) constituted the most common research methods used by authors, as presented in Figure $2 \mathrm{~b}$. Among the empirical studies, most of the papers used a cross-sectional design. However, none of these studies tracked the evolution of barriers and enablers over time. Barriers and enablers faced by SMEs have mainly been studied in the literature considering the environmental perspective of sustainability, Figure 2c, with $69 \%$ of the papers considering barriers and enablers associated with the adoption of environmental sustainability practices, whereas only $16 \%$ of papers in the sample presented barriers and enablers from a triple bottom line perspective. 


\subsection{Barriers and Enablers for the Adoption of Sustainable Manufacturing}

The content analysis identified 61 barriers and 35 enablers (described in detail in Appendix A Table A1). The categorization of barriers and enablers resulted in the following seven main categories:

(a) Organizational, managerial, and attitudinal aspects (OMA) (related to managerial skills, organizational culture and structure, and attitudes and beliefs).

(b) Informational aspects (Inf) (related to information and knowledge).

(c) Governmental aspects (Gov) (related to factors emerging from governmental policy).

(d) Financial aspects (F) (related to aspects of economic or financial nature affecting the longevity of the enterprise).

(e) Training and skills development aspects (TSD) (related to receiving or giving instruction, training programs in order to ensure human resources have the necessary skills and knowledge).

(f) Market and business context aspects (M) (related to SMEs' relations with suppliers, customers, and other stakeholders within the business context that prevent or facilitate the adoption of sustainable manufacturing).

(g) Technological aspects (T) (related to the machinery, equipment, devices, and technology).

\subsubsection{Barriers and Enablers per Category}

Figure $3 a, b$, respectively, show the distribution of barriers and enablers sorted by category. The OMA category contained the largest number of barriers (22), followed by the governmental category (11) and the informational category (10). The training and skill development category contained only three barriers. Regarding the distribution of the enablers, the OMA category also contained the largest number of enablers (14), followed by the governmental category (9), and the market and business context category (5). The training and skill development category contained a single enabler, and the technological category contained none.

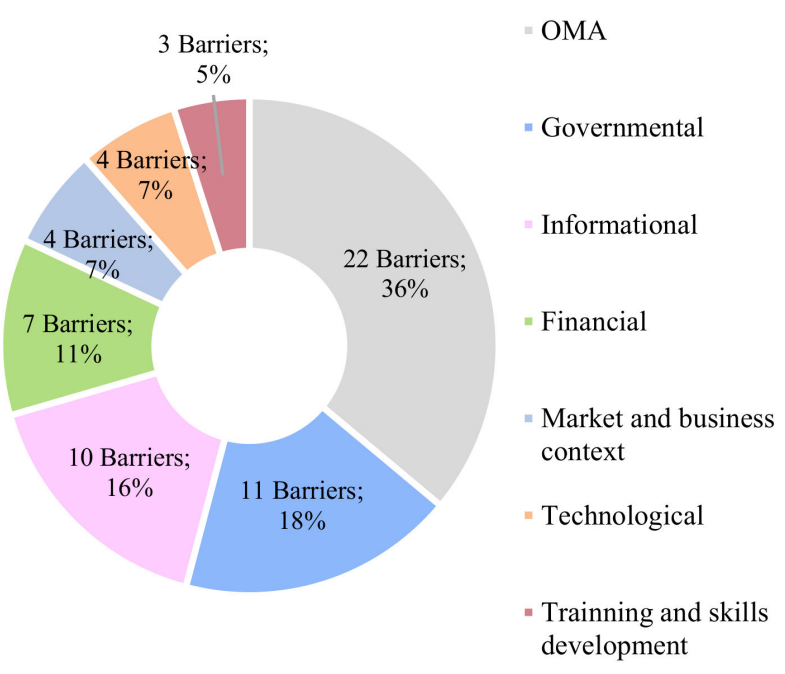

(a)

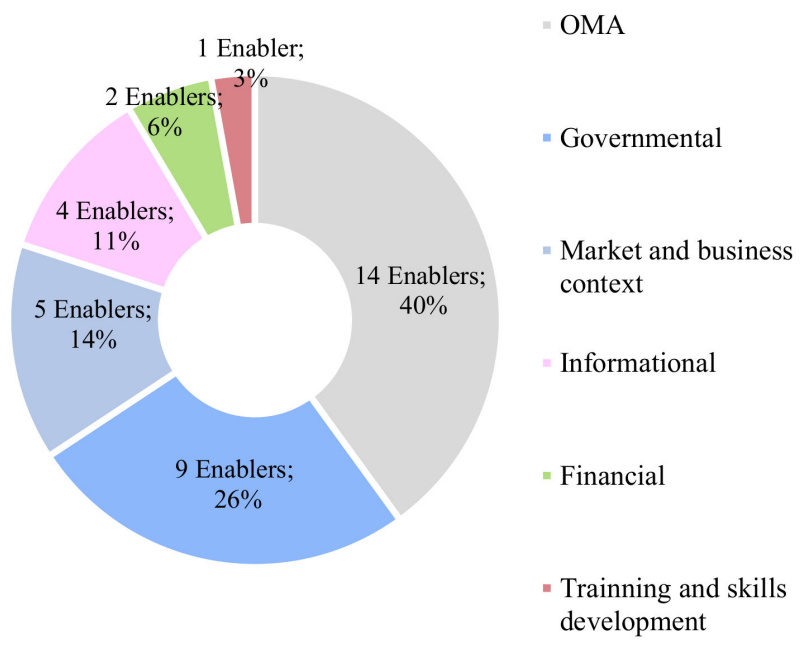

(b)

Figure 3. Distribution of barriers (a) and enablers (b) per category within the final sample.

Some barriers and enablers appeared in many of the papers included in the final sample, whereas some only a few times. Table 2 shows the most and least frequently mentioned barriers and enablers, i.e., those mentioned in more than $31 \%$ of the papers and less than $3 \%$ of the papers, respectively. Individual frequency values are indicated in the detailed compilation of barriers and enablers in Appendix A Table A1. This study showed that the most frequently mentioned barriers in the literature correspond belonged to the OMA and financial categories, while the most frequently mentioned enablers are equally 
distributed among the categories. The frequencies of the most highly mentioned barriers and enablers are presented in Appendix A Figures A1 and A2.

Table 2. Frequency of barriers and enablers from the final sample of reviewed papers.

\begin{tabular}{|c|c|c|}
\hline & Barriers & Enablers \\
\hline $\begin{array}{l}\text { Most frequently } \\
\text { mentioned }\end{array}$ & $\begin{array}{l}\text { Lack of access to external technical } \\
\text { knowledge to adopt sustainable } \\
\text { manufacturing practices (B23); low } \\
\text { skilled labor (B59); lack of awareness of } \\
\text { the benefits derived from sustainable } \\
\text { manufacturing practices (B16); lack of } \\
\text { financial resources (B43); manager's } \\
\text { misunderstandings and pessimistic } \\
\text { preconceptions (B2); lack of time for } \\
\text { planning, execution and review of } \\
\text { sustainable manufacturing practices } \\
\text { (B9); little knowledge on sustainability } \\
\text { in SMEs (B24); difficulties accessing } \\
\text { financial capital (B44); resistance to } \\
\text { change (B18), lack of effective } \\
\text { legislation and/or weak regulatory } \\
\text { environment (B37); high investment } \\
\text { costs and low returns of } \\
\text { environmentally technologies (B45). }\end{array}$ & $\begin{array}{l}\text { Knowledge networks and social } \\
\text { networks (E6); provision of } \\
\text { financial incentives to foster } \\
\text { sustainable manufacturing (E17); } \\
\text { pressure from market and clients } \\
\text { (E24); government regulations } \\
\text { fostering sustainability adoption } \\
\text { (E20). }\end{array}$ \\
\hline $\begin{array}{l}\text { Least frequently } \\
\text { mentioned }\end{array}$ & $\begin{array}{l}\text { Concerns that environmental } \\
\text { investments undermine } \\
\text { competitiveness (B11); certain subsidies } \\
\text { impeding sustainable manufacturing } \\
\text { (B35); low political participation of } \\
\text { SMEs (B42); difficulty quantifying the } \\
\text { financial performance of environmental } \\
\text { investments (B48); lack of awareness on } \\
\text { international trends related to } \\
\text { environmental sustainability (B50); lack } \\
\text { of awareness of SMEs role on social } \\
\text { capital (B53). }\end{array}$ & $\begin{array}{l}\text { Managerial support toward } \\
\text { collaborative environment for } \\
\text { innovation (E2); develop } \\
\text { continuously and quick } \\
\text { adaptation (E5); adoption of lean } \\
\text { manufacturing (E8); more efficient } \\
\text { routines and practices (E10), use } \\
\text { of business analysis tools (E12); } \\
\text { availability of flexible and } \\
\text { innovative human resources (E13); } \\
\text { flat management structure and } \\
\text { empowerment of workforce (E14); } \\
\text { institutionalize rewards } \\
\text { recognizing adoption of } \\
\text { sustainable manufacturing in } \\
\text { SMEs (E23). }\end{array}$ \\
\hline
\end{tabular}

4.2.2. Barriers and Enablers for Sustainable Manufacturing Lacking an Integrative Perspective

Most of the identified barriers were related to specific sustainable manufacturing practice (e.g., barriers to adopting life cycle assessment and barriers to industrial energy efficiency in SMEs), compared to a significant smaller number of barriers toward more sustainability integrated approaches (e.g., barriers and enablers for sustainable manufacturing, barriers for clean production and barriers to engaging in corporate social responsibility). Similarly, most of the identified enablers were related to a specific sustainable manufacturing practice (e.g., enablers and barriers for adopting environmental management systems), compared to the papers addressing enablers from more integrated sustainability approaches (e.g., characteristics for achieving sustainable manufacturing companies). Exemplifying this, and from a more integrated perspective, Coppa and Sriramesh [63] explored the enablers for SMEs to engage in CSR practices, and found that social networks consolidate the strong relationships among stakeholders that constitute the social capital of SMEs, which is closely associated with an increase in organizational competitiveness and, thus, the enterprise's longevity. Among the few papers indicating enablers for improving the 
economic sustainability of SMEs, Thomas et al. [64] proposed a set of factors for the development of economically sustainable manufacturing SMEs, concluding that SMEs aiming for business sustainability should focus on achieving business growth while reducing resource consumption, both in manufacturing processes and final products. Lean implementation was highlighted as an enabler for reducing operating costs. When combined with product innovation strategies, lean implementation would enable SMEs to break into new markets and, thus, become resilient to market changes and capable of attaining long-term economic sustainability.

\subsubsection{Internal and External Barriers and Enablers}

Previous studies have stressed the importance of indicating the internal or external nature of barriers and enablers [44]. The internal or external nature of a factor is dictated by its origin with respect to the SMEs and by the ability of SMEs to influence the factor [34-36]. Very few studies in our sample indicated the internal and external characteristic of the factors $[21,36,37,43,65]$. The systematic review of the literature carried out in this paper revealed 61 barriers and 35 enablers (See Appendix A Table A1). Most of the identified barriers were internal (51\%), while the remaining (49\%) were external. Regarding the enablers, the majority were found to be external (51\%), while the remaining (49\%) had an internal origin. An overview of the identified barriers and enablers is presented in Figure 4. External factors are followed by (E) and displayed in a darker shade compared to the internal ones (followed by (I)).
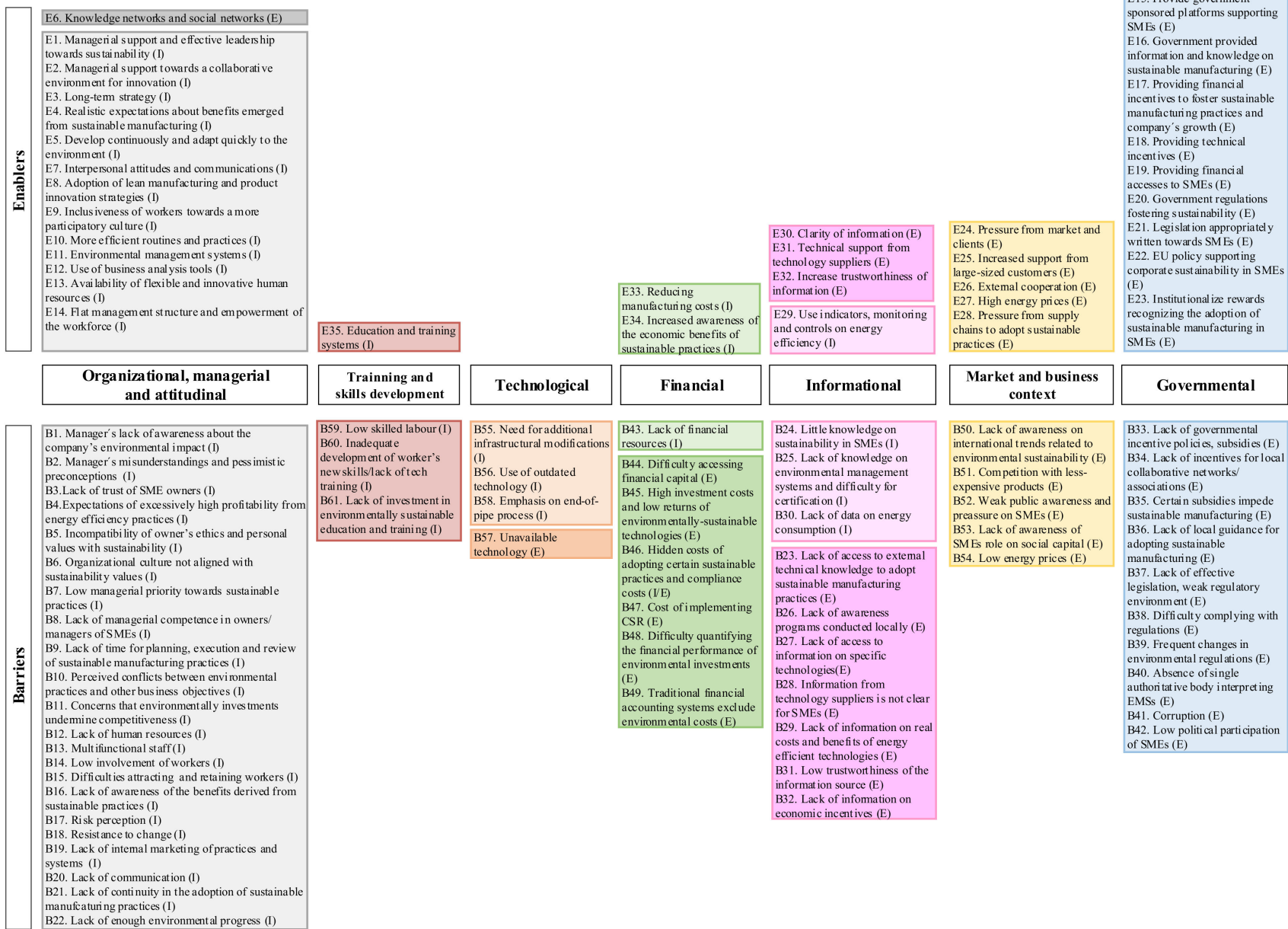

Figure 4. Schematic presentation of the identified barriers and enablers for the adoption of sustainable manufacturing in manufacturing SMEs. 


\subsection{Mitigating Barriers through Enablers for Sustainable Manufacturing}

The second research question in this paper was related to what enabler might be used to mitigate the barriers. With this question in mind, Table 3 presents the barriers alongside the enablers with the potential to mitigate them. ' $\mathrm{I}$ ' indicates the enabler is internal, while ' $E$ ' indicates it is external. Some enablers address specific barriers more directly or are expected to have a more direct mitigating effect on a barrier than others. Table 3 indicates such enablers using the symbol $\diamond$.

Table 3. Identified barriers matched with enablers that could mitigate them.

\begin{tabular}{|c|c|c|}
\hline Cat. & Barriers & Enablers Mitigating Specific Barriers \\
\hline \multirow{29}{*}{$\begin{array}{l}\text { Organizational, } \\
\text { managerial and } \\
\text { attitudinal } \\
\text { (OMA) }\end{array}$} & \multirow{6}{*}{$\begin{array}{l}\text { B1. Manager's lack of awareness } \\
\text { about the company's environmental } \\
\text { sustainability impact (I) }\end{array}$} & $\diamond \mathrm{E} 12$. Use of business analysis tools (I/OMA) \\
\hline & & $\triangleright$ E16. Government provided information on sustainable manufacturing (E/Gov) \\
\hline & & $\diamond$ E11. Environmental management systems (I/OMA) \\
\hline & & E15. Provide government sponsored platforms supporting SMEs (E/Gov) \\
\hline & & E6. Knowledge networks and social networks (E/OMA) \\
\hline & & E26. External cooperation (E/M) \\
\hline & \multirow{4}{*}{$\begin{array}{l}\text { B2. Manager's misunderstandings } \\
\text { and pessimistic preconceptions (I) }\end{array}$} & $\begin{array}{l}\checkmark \text { E4. Realistic expectations about benefits emerged from sustainable manufacturing } \\
\text { (I/OMA) }\end{array}$ \\
\hline & & E30. Clarity of information (E/Inf) \\
\hline & & E15. Provide government sponsored platforms supporting SMEs (E/Gov) \\
\hline & & $\begin{array}{l}\text { E28. Pressure from supply chains to adopt sustainable manufacturing } \\
\text { Practices (E/M) }\end{array}$ \\
\hline & B3. Lack of trust of SME owners (I) & E6. Knowledge networks and social networks (E/OMA) \\
\hline & \multirow{2}{*}{$\begin{array}{l}\text { B4. Expectations of excessively high } \\
\text { profitability from energy efficiency } \\
\text { practices (I) }\end{array}$} & $\begin{array}{l}\checkmark \text { E4. Realistic expectations about benefits emerged from sustainable manufacturing } \\
\text { (I/OMA) }\end{array}$ \\
\hline & & E30. Clarity of information (E/Inf) \\
\hline & $\begin{array}{l}\text { B5. Incompatibility of owner's } \\
\text { ethics and personal values with } \\
\text { sustainability (I) }\end{array}$ & $\diamond$ E1. Managerial support and effective leadership toward sustainability (I/OMA) \\
\hline & \multirow{3}{*}{$\begin{array}{l}\text { B6. Organizational culture not } \\
\text { aligned with sustainability values (I) }\end{array}$} & E1. Managerial support and effective leadership toward sustainability (I/OMA) \\
\hline & & E14. Flat management structure and empowerment of workforce (I/OMA) \\
\hline & & E11. Environmental management systems (I/OMA) \\
\hline & \multirow{6}{*}{$\begin{array}{l}\text { B7. Low managerial priority toward } \\
\text { sustainable manufacturing } \\
\text { practices (I) }\end{array}$} & $\checkmark$ E1. Managerial support and effective leadership toward sustainability (I/OMA) \\
\hline & & $\diamond \mathrm{E} 12$. Use of business analysis tools (I/OMA) \\
\hline & & E15. Provide government sponsored platforms supporting SMEs (E/Gov) \\
\hline & & $\begin{array}{l}\text { E28. Pressure from supply chains to adopt sustainable manufacturing } \\
\text { practices(E/M) }\end{array}$ \\
\hline & & E24. Pressure from market and clients (E/M) \\
\hline & & $\begin{array}{l}\text { E23. Institutionalize rewards recognizing the adoption of sustainable } \\
\text { manufacturing in SMEs (E/Gov) }\end{array}$ \\
\hline & \multirow{6}{*}{$\begin{array}{l}\text { B8. Lack of managerial competence } \\
\text { in owners/managers of SMEs (I) }\end{array}$} & E15. Provide government sponsored platforms supporting SMEs (E/Gov) \\
\hline & & E16. Government provided information on sustainable manufacturing (E/Gov) \\
\hline & & E35. Education and training systems to improve operations (I/TSD) \\
\hline & & E11. Environmental management systems (I/OMA) \\
\hline & & E26. External cooperation (E/M) \\
\hline & & E6. Knowledge networks and social networks (E/OMA) \\
\hline
\end{tabular}


Table 3. Cont.

\begin{tabular}{|c|c|c|}
\hline Cat. & Barriers & Enablers Mitigating Specific Barriers \\
\hline \multirow{35}{*}{$\begin{array}{l}\text { Organizational, } \\
\text { managerial and } \\
\text { attitudinal } \\
\text { (OMA) }\end{array}$} & $\begin{array}{l}\text { B9. Lack of time for planning, } \\
\text { execution and review of sustainable } \\
\text { practices (I) }\end{array}$ & E13. Availability of flexible and innovative human resources (I/OMA) \\
\hline & \multirow{2}{*}{$\begin{array}{l}\text { B10. Perceived conflicts between } \\
\text { environmental practices and other } \\
\text { business objectives (I) }\end{array}$} & $\diamond \mathrm{E} 12$. Use of business analysis tools (I/OMA) \\
\hline & & E1. Managerial support and effective leadership toward sustainability (I/OMA) \\
\hline & \multirow{5}{*}{$\begin{array}{l}\text { B11. Concerns that environmental } \\
\text { investments undermine } \\
\text { competitiveness (I) }\end{array}$} & $\begin{array}{l}\checkmark \text { E4. Realistic expectations about benefits emerged from sustainable manufacturing } \\
\text { (I/OMA) }\end{array}$ \\
\hline & & $\diamond \mathrm{E} 12$. Use of business analysis tools (I/OMA) \\
\hline & & E8. Adoption of lean manufacturing and product innovation strategies (I/OMA) \\
\hline & & E26. External cooperation (E/M) \\
\hline & & E6. Knowledge networks and social networks (E/OMA) \\
\hline & \multirow{2}{*}{ B12. Lack of human resources (I) } & $\diamond$ E13. Availability of flexible and innovative human resources (I/OMA) \\
\hline & & E6. Knowledge networks and social networks (E/OMA) \\
\hline & B13. Multifunctional staff (I) & $\diamond$ E13. Availability of flexible and innovative human resources (I/OMA) \\
\hline & $\begin{array}{l}\text { B14. Low involvement of } \\
\text { workers (I) }\end{array}$ & E9. Inclusiveness of workers toward a more participatory culture (I/OMA) \\
\hline & \multirow{5}{*}{$\begin{array}{l}\text { B15. Difficulties attracting and } \\
\text { retaining workers }(\mathrm{I})\end{array}$} & E9. Inclusiveness of workers toward a more participatory culture (I/OMA) \\
\hline & & E14. Flat management structure and empowerment of workforce (I/OMA) \\
\hline & & E35. Education and training systems to improve operations (I/TSD) \\
\hline & & E7. Interpersonal attitudes and communications (I/OMA) \\
\hline & & E11. Environmental management systems (I/OMA) \\
\hline & \multirow{6}{*}{$\begin{array}{l}\text { B16. Lack of awareness of the } \\
\text { benefits derived from sustainable } \\
\text { manufacturing practices (I) }\end{array}$} & $\checkmark$ E35. Education and training systems to improve operations (I/TSD) \\
\hline & & $\diamond$ E11. Environmental management systems (I/OMA) \\
\hline & & $\diamond$ E6. Knowledge networks and social networks (E/OMA) \\
\hline & & E15. Provide government sponsored platforms supporting SMEs (E/Gov) \\
\hline & & E16. Government provided information on sustainable manufacturing (E/Gov) \\
\hline & & $\begin{array}{l}\text { E28. Pressure from supply chains to adopt sustainable manufacturing } \\
\text { Practices (E/M) }\end{array}$ \\
\hline & \multirow{4}{*}{ B17. Risk perception (I) } & E30. Clarity of information (E/Inf) \\
\hline & & E15. Provide government sponsored platforms supporting SMEs (E/Gov) \\
\hline & & E16. Government provided information on sustainable manufacturing (E/Gov) \\
\hline & & E25. Increased support from large customers (E/M) \\
\hline & \multirow{6}{*}{ B18. Resistance to change (I) } & $\diamond \mathrm{E} 2$. Managerial support for collaborative environment for innovation (I/OMA) \\
\hline & & $\diamond$ E5. Develop continuously/adapt to the environment (I/OMA) \\
\hline & & E11. Environmental management systems (I/OMA) \\
\hline & & E8. Adoption of lean manufacturing and product innovation strategies (I/OMA) \\
\hline & & $\begin{array}{l}\text { E28. Pressure from supply chains to adopt sustainable manufacturing } \\
\text { practices }(\mathrm{E} / \mathrm{M})\end{array}$ \\
\hline & & E25. Increased support from large customers (E/M) \\
\hline & \multirow{2}{*}{$\begin{array}{l}\text { B19. Lack of internal marketing of } \\
\text { practices and systems (I) }\end{array}$} & E35. Education and training systems to improve operations (I/TSD) \\
\hline & & E11. Environmental management systems (I/OMA) \\
\hline
\end{tabular}


Table 3. Cont.

\begin{tabular}{|c|c|c|}
\hline Cat. & Barriers & Enablers Mitigating Specific Barriers \\
\hline \multirow{13}{*}{$\begin{array}{l}\text { Organizational, } \\
\text { managerial and } \\
\text { attitudinal } \\
\text { (OMA) }\end{array}$} & \multirow{3}{*}{ B20. Lack or poor communication (I) } & $\diamond \mathrm{E} 7$. Interpersonal attitudes and communications (I/OMA) \\
\hline & & E11. Environmental management systems (I/OMA) \\
\hline & & E6. Knowledge networks and social networks (E/OMA) \\
\hline & \multirow{7}{*}{$\begin{array}{l}\text { B21. Lack of continuity in the } \\
\text { adoption of sustainable } \\
\text { manufacturing practices (I) }\end{array}$} & $\diamond$ E11. Environmental management systems (I/OMA) \\
\hline & & $\diamond$ E3. Long-term strategy (I/OMA) \\
\hline & & $\diamond \mathrm{E} 25$. Increased support from large customers $(\mathrm{E} / \mathrm{M})$ \\
\hline & & $\diamond$ E1. Managerial support and effective leadership toward sustainability (I/OMA) \\
\hline & & E28. Pressure from supply chains to adopt sustainable manufacturing (E/M) \\
\hline & & E5. Develop continuously/adapt to the environment(I/OMA) \\
\hline & & E6. Knowledge networks and social networks (E/OMA) \\
\hline & \multirow{3}{*}{$\begin{array}{l}\text { B22. Lack of enough environmental } \\
\text { progress (I) }\end{array}$} & $\begin{array}{l}\vee \text { E4. Realistic expectations about benefits emerged from sustainable } \\
\text { manufacturing (I/OMA) }\end{array}$ \\
\hline & & E6. Knowledge networks and social networks (E/OMA) \\
\hline & & E25. Increased support from large customers (E/M) \\
\hline \multirow{29}{*}{ Informational (Inf) } & \multirow{6}{*}{$\begin{array}{l}\text { B23. Lack of access to external } \\
\text { technical knowledge to adopt } \\
\text { sustainable manufacturing } \\
\text { practices (E) }\end{array}$} & $\diamond$ E16. Government provided information on sustainable manufacturing (E/Gov) \\
\hline & & $\diamond$ E15. Provide government sponsored platforms supporting SMEs (E/Gov) \\
\hline & & $\diamond \mathrm{E} 31$. Technical support from technology suppliers (E/Inf) \\
\hline & & E6. Knowledge networks and social networks (E/OMA) \\
\hline & & E26. External cooperation (E/M) \\
\hline & & E25. Increased support from large customers (E/M) \\
\hline & \multirow{5}{*}{$\begin{array}{l}\text { B24. Little knowledge on } \\
\text { sustainability in SMEs (I) }\end{array}$} & $\diamond$ E15. Provide government sponsored platforms supporting SMEs (E/Gov) \\
\hline & & $\diamond$ E16. Government provided information on sustainable manufacturing (E/Gov) \\
\hline & & E6. Knowledge networks and social networks (E/OMA) \\
\hline & & E25. Increased support from large customers (E/M) \\
\hline & & E26. External cooperation (E/M) \\
\hline & \multirow{4}{*}{$\begin{array}{l}\text { B25. Lack of knowledge on } \\
\text { environmental systems and } \\
\text { difficulty for certification (I) }\end{array}$} & E15. Provide government sponsored platforms supporting SMEs (E/Gov) \\
\hline & & E6. Knowledge networks and social networks (E/OMA) \\
\hline & & E25. Increased support from large customers (E/M) \\
\hline & & E26. External cooperation (E/M) \\
\hline & \multirow{3}{*}{$\begin{array}{l}\text { B26. Lack of awareness programs } \\
\text { conducted locally (E) }\end{array}$} & $\diamond$ E6. Knowledge networks and social networks (E/OMA) \\
\hline & & $\diamond$ E26. External cooperation $(\mathrm{E} / \mathrm{M})$ \\
\hline & & E15. Provide government sponsored platforms supporting SMEs (E Gov) \\
\hline & \multirow{2}{*}{$\begin{array}{l}\text { B27. Lack of access to information } \\
\text { specific technologies (E) }\end{array}$} & E6. Knowledge networks and social networks (E/OMA) \\
\hline & & E26. External cooperation (E/M) \\
\hline & \multirow{3}{*}{$\begin{array}{l}\text { B28. Information from technology } \\
\text { suppliers is not clear for SMEs }(\mathrm{E})\end{array}$} & $\diamond \mathrm{E} 30$. Clarity of information (E/Inf) \\
\hline & & E6. Knowledge networks and social networks (E/OMA) \\
\hline & & E26. External cooperation (E/M) \\
\hline & \multirow{2}{*}{$\begin{array}{l}\text { B29. Lack of information on real } \\
\text { costs and benefits of energy efficient } \\
\text { technologies (E) }\end{array}$} & E32. Increase trustworthiness information(E/Inf) \\
\hline & & E6. Knowledge networks and social networks (E/OMA) \\
\hline & $\begin{array}{l}\text { B30. Lack of data on energy } \\
\text { consumption }\end{array}$ & $\diamond \mathrm{E} 29$. Use indicators, monitoring and controls on energy efficiency \\
\hline & $\begin{array}{l}\text { B31. Low trustworthiness of the } \\
\text { information source }(E)\end{array}$ & $\diamond \mathrm{E} 32$. Increase trustworthiness information (E/Inf) \\
\hline & \multirow{2}{*}{$\begin{array}{l}\text { B32. Lack of information on } \\
\text { economic incentives }(E)\end{array}$} & $\begin{array}{l}\diamond \text { E17. Provide financial incentives to foster sustainable manufacturing practices } \\
\text { and company's growth (E Gov) }\end{array}$ \\
\hline & & E26. External cooperation (E/M) \\
\hline
\end{tabular}


Table 3. Cont.

\begin{tabular}{|c|c|c|}
\hline Cat. & Barriers & Enablers Mitigating Specific Barriers \\
\hline \multirow{14}{*}{ Governmental (G) } & \multirow{2}{*}{$\begin{array}{l}\text { B33. Lack of governmental incentive } \\
\text { policies, subsidies (E) }\end{array}$} & $\diamond$ E18. Provide technical incentives (E/Gov) \\
\hline & & $\begin{array}{l}\diamond \text { E17. Provide financial incentives to foster sustainable manufacturing practices } \\
\text { and company's growth (E/Gov) }\end{array}$ \\
\hline & \multirow{2}{*}{$\begin{array}{l}\text { B34. Lack of incentives for local } \\
\text { collaborative networks / } \\
\text { associations (E) }\end{array}$} & $\diamond$ E6. Knowledge networks and social networks (E/OMA) \\
\hline & & $\diamond \mathrm{E} 26$. External cooperation $(\mathrm{E} / \mathrm{M})$ \\
\hline & $\begin{array}{l}\text { B35. Certain subsidies that impede } \\
\text { sustainable manufacturing (E) }\end{array}$ & $\begin{array}{l}\diamond \text { E17. Provide financial incentives to foster sustainable manufacturing practices } \\
\text { and company's growth (E/Gov) }\end{array}$ \\
\hline & \multirow{3}{*}{$\begin{array}{l}\text { B36. Lack of local guidance for } \\
\text { adopting sustainable } \\
\text { manufacturing (E) }\end{array}$} & $\diamond$ E15. Provide government sponsored platforms supporting SMEs (E/Gov) \\
\hline & & $\diamond$ E16. Government provided information on sustainable manufacturing (E/Gov) \\
\hline & & E25. Increased support from large customers (E/M) \\
\hline & $\begin{array}{l}\text { B37. Lack of effective legislation, } \\
\text { weak regulatory environment }(\mathrm{E})\end{array}$ & $\diamond \mathrm{E} 20$. Government regulations fostering sustainability adoption (E/Gov) \\
\hline & $\begin{array}{l}\text { B38. Difficulty complying with } \\
\text { regulations (E) }\end{array}$ & $\diamond$ E21. Legislation appropriately written toward SMEs (E/Gov) \\
\hline & $\begin{array}{l}\text { B39. Frequent changes in } \\
\text { environmental regulations (E) }\end{array}$ & - \\
\hline & $\begin{array}{l}\text { B40. Absence of single authoritative } \\
\text { body interpreting EMSs (E) }\end{array}$ & - \\
\hline & B41. Corruption (E) & - \\
\hline & $\begin{array}{l}\text { B42. Low political participation of } \\
\text { SMEs (E) }\end{array}$ & E22. EU policy supporting corporate sustainability in SMEs (E/Gov) \\
\hline \multirow{12}{*}{ Financial (F) } & \multirow{2}{*}{ B43. Lack of financial resources (I) } & $\diamond$ E33. Reducing manufacturing costs (I/F) \\
\hline & & E19. Provide financial accesses to SMEs (E/Gov) \\
\hline & $\begin{array}{l}\text { B44. Difficulty accessing financial } \\
\text { capital (E) }\end{array}$ & $\diamond$ E19. Provide financial accesses to SMEs (E/Gov) \\
\hline & \multirow{2}{*}{$\begin{array}{l}\text { B45. High investment costs and low } \\
\text { returns of environmentally- } \\
\text { sustainable technologies }(\mathrm{E})\end{array}$} & E6. Knowledge networks and social networks (E/OMA) \\
\hline & & E25. Increased support from large customers (E/M) \\
\hline & \multirow{3}{*}{$\begin{array}{l}\text { B46. Hidden costs of adopting } \\
\text { certain sustainable manufacturing } \\
\text { practices and compliance costs (I/E) }\end{array}$} & $\begin{array}{l}\checkmark \text { E34. Increased awareness of the economic benefits of sustainable manufacturing } \\
\text { practices (I/F) }\end{array}$ \\
\hline & & $\diamond$ E33. Reducing manufacturing costs $(\mathrm{I} / \mathrm{F})$ \\
\hline & & E25. Increased support from large customers (E/M) \\
\hline & \multirow[t]{2}{*}{ B47. Cost of implementing CSR (E) } & $\begin{array}{l}\diamond \text { E34. Increased awareness of the economic benefits of sustainable manufacturing } \\
\text { practices }(\mathrm{I} / \mathrm{F})\end{array}$ \\
\hline & & $\diamond$ E33. Reducing manufacturing costs (I/F) \\
\hline & $\begin{array}{l}\text { B48. Difficulty quantifying the } \\
\text { financial performance of } \\
\text { environmental investments (E) }\end{array}$ & - \\
\hline & $\begin{array}{l}\text { B49. Traditional financial } \\
\text { accountings systems exclude } \\
\text { environmental costs (E) }\end{array}$ & - \\
\hline \multirow{9}{*}{$\begin{array}{l}\text { Market and } \\
\text { business } \\
\text { context }(\mathrm{M})\end{array}$} & \multirow{4}{*}{$\begin{array}{l}\text { B50. Lack of awareness of } \\
\text { international trends related to } \\
\text { environmental sustainability (E) }\end{array}$} & E6. Knowledge networks and social networks (E/OMA) \\
\hline & & E24. Pressure from market and clients (E/M) \\
\hline & & E25. Increased support from large customers (E/M) \\
\hline & & E26. External cooperation (E/M) \\
\hline & \multirow{2}{*}{$\begin{array}{l}\text { B51. Competition with } \\
\text { less-expensive products }(E)\end{array}$} & E8. Adoption of lean manufacturing and product innovation strategies (I/OMA) \\
\hline & & E25. Increased support from large customers (E/M) \\
\hline & $\begin{array}{l}\text { B52. Weak public awareness and } \\
\text { pressure on SMEs (E) }\end{array}$ & $\diamond \mathrm{E} 24$. Pressure from market and clients $(\mathrm{E} / \mathrm{M})$ \\
\hline & $\begin{array}{l}\text { B53. Lack of awareness of SMEs role } \\
\text { on social capital (E) }\end{array}$ & $\triangleright$ E6. Knowledge networks and social networks (E/OMA) \\
\hline & B54. Low energy prices (E) & $\diamond E 27$. High energy prices $(\mathrm{E} / \mathrm{M})$ \\
\hline
\end{tabular}


Table 3. Cont.

\begin{tabular}{|c|c|c|}
\hline Cat. & Barriers & Enablers Mitigating Specific Barriers \\
\hline \multirow{7}{*}{ Technological (T) } & $\begin{array}{l}\text { B55. Need for additional } \\
\text { infrastructural modifications (I) }\end{array}$ & $\diamond \mathrm{E} 10$. More efficient routines and practices (I/OMA) \\
\hline & B56. Use of outdated technology (I) & $\diamond$ E18. Provide technical incentives (E/Gov) \\
\hline & B57. Unavailable technology (E) & $\checkmark$ E18. Provide technical incentives (E/Gov) \\
\hline & \multirow{4}{*}{$\begin{array}{l}\text { B58. Emphasis on end-of-pipe } \\
\text { process (I) }\end{array}$} & E20. Government regulations fostering sustainability adoption (E/Gov) \\
\hline & & $\begin{array}{l}\text { E17. Provide financial incentives to foster sustainable manufacturing practices } \\
\text { and company's growth (E Gov) }\end{array}$ \\
\hline & & E24. Pressure from market and clients (E/M) \\
\hline & & $\begin{array}{l}\text { E28. Pressure from supply chains to adopt sustainable manufacturing practices } \\
\text { (E/M) }\end{array}$ \\
\hline \multirow{5}{*}{$\begin{array}{l}\text { Training and skill } \\
\text { development } \\
\text { (TSD) }\end{array}$} & \multirow{2}{*}{ B59. Low skilled labor (I) } & $\diamond$ E35. Education and training systems to improve operations (I/TSD) \\
\hline & & E6. Knowledge networks and social networks (E/OMA) \\
\hline & \multirow{2}{*}{$\begin{array}{l}\text { B60. Inadequate development of } \\
\text { worker's new skills/lack of tech } \\
\text { training (I) }\end{array}$} & $\checkmark$ E35. Education and training systems to improve operations (I/TSD) \\
\hline & & E6. Knowledge networks and social networks (E/OMA) \\
\hline & $\begin{array}{l}\text { B61. Lack of investment in } \\
\text { environmentally sustainable } \\
\text { education and training (I) }\end{array}$ & E1. Managerial support and effective leadership toward sustainability (I/OMA) \\
\hline
\end{tabular}

As can be noted in Table 3, not all barriers could be addressed by the enablers extracted from the literature: difficulty quantifying the financial performance of environmental investments (B48); traditional financial accounting systems exclude environmental costs (B49); frequent changes in environmental regulations (B39); absence of single authoritative body interpreting EMSs (B40); and corruption (B41). In addition, the distribution of enablers from other categories expected to mitigate barriers within a given category is presented in Appendix A Figure A3.

This study found that there is a high occurrence of OMA enablers expected to overcome the identified barriers, followed by market and business context and governmental enablers (see Table 3, Figures 4 and 5, and Appendix A Figure A3). Therefore, the enablers belonging to those categories most often contributed partially or directly to the mitigation of barriers.

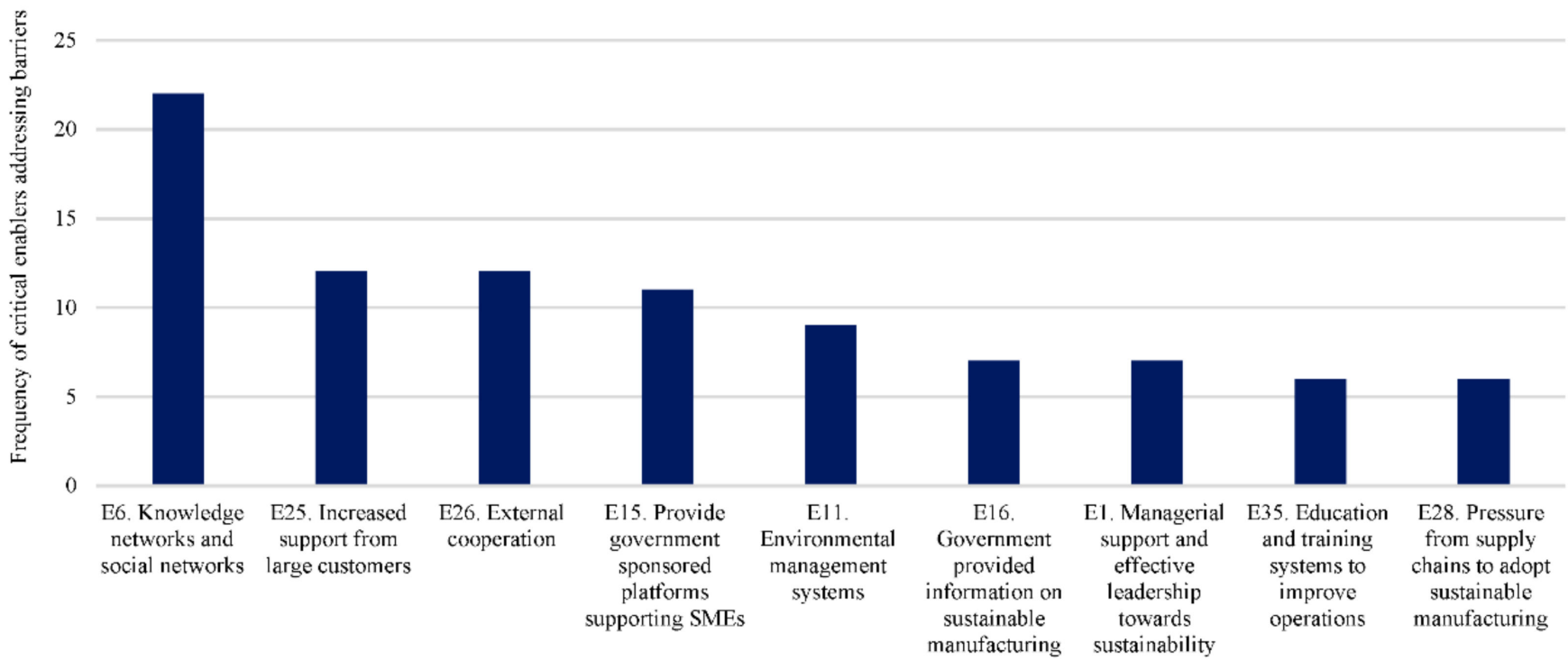

Figure 5. Critical enablers expected to address the highest number of identified barriers. 
Lastly, the "critical enablers" according to the frequency with which they were expected to address the identified barriers are presented in Figure 5. The critical enablers belonged in its majority to the OMA, as well as market and business context categories (knowledge networks and social networks (E6) being the top critical enabler).

\section{Discussion}

This section comprises a two-fold discussion: firstly, it discusses the identified barriers and enablers faced by manufacturing SMEs when adopting sustainable manufacturing practices; secondly, it discusses what identified enablers that could contribute to mitigate the identified barriers.

\subsection{Barriers and Enablers for Sustainable Manufacturing in SMEs}

The systematic literature review revealed that researchers have studied barriers to a higher extent compared to the enablers. This predominance of barriers over enablers is consistent with previous findings $[19,23]$. Our study showed that the most commonly mentioned barriers in the literature made reference to lack of access to external technical knowledge (B23), low skilled labor (B59), lack of awareness of the benefits from sustainable manufacturing practices (B16), and lack of financial resources (B43).

Regarding the enablers identified from the review, knowledge, and social networks (E6), was the most commonly mentioned. Knowledge networks (E6) can mitigate the following barriers: lack of access to external technical knowledge (B23); low skilled labor (B59); and little knowledge about sustainability (B24). However, knowledge networks (E6) also facilitate the development of social capital by SMEs through mutual relationships with other SMEs and collaboration in formal or informal networks, which can lead to improved SME social performance. Thus, knowledge networks constitute a crucial enabler for SMEs to improve knowledge transfer from stakeholders to SMEs and to promote technology development, thereby enabling SMEs to gain the knowledge that is essential to the successful adoption of sustainable manufacturing practices. The provision of financial incentives to foster sustainable manufacturing (E17) constituted another commonly mentioned enabler. Financial incentives are commonly recognized for promoting sustainable manufacturing practices and company growth. Subsidies are critical for the adoption of sustainable manufacturing practices as they help steer investments toward technologies and manufacturing practices leading to improved sustainability performance. Similarly, tax deductions and depreciation allowances might also provide incentives for SMEs to change inefficient equipment more quickly [66].

The categorization, that resulted from an inductive content analysis of the final sample of papers in the literature, was found to be similar to categorizations developed in earlier studies [22-24]. Shi et al. [22] categorized the barriers to implementing clean manufacturing according to: policy and market barriers, financial and economic barriers, technical and information barriers, and organizational and managerial barriers. Henriquez and Catarino [23] divided the barriers to improving energy efficiency into eight categories: organizational, management, financing, government, economic, training and knowledge, behavioral, and technical. Similarly, Oliveira Neto et al. [24] grouped the barriers to implementing clean production into six main categories: economic and financial, technological, cultural, legislation, government, and organizational. Most of these studies did not explain the reasons for their respective categorizations or describe the factors (enabler or barriers) within their categories. The categories mentioned in these earlier studies typically refer to organizational, financial, technological, market, and government factors. Our categories include separate training and skills development and informational categories (as well as the ones similar to those referred in previous studies), whereas many enablers and barriers in these two new categories have previously been classified as belonging to the organizational or technological categories. Separating the training and skills development and informational categories permits increased understanding of individual factors related to skills development and informational aspects of adopting sustainable manufacturing 
practices and illuminates the relationships between the factors in these new categories that may not be obvious when they are viewed as factors within broader organizational or technological categories.

In our categorization, the OMA category contained the highest number of identified barriers and enablers. Previous studies have emphasized the importance of management and human resources when adopting sustainable manufacturing practices [21,67]. Given that SMEs are commonly characterized by low effectiveness in transferring managerial knowledge and practices, many barriers faced by SMEs relate to managerial competencies and management practices that, at the end, represent obstacles when trying to adopt sustainable manufacturing practices and to remain profitable over time.

The governmental category contained the second largest number of barriers and enablers, confirming previous findings pointing out that environmental legislation compliance is more difficult for SMEs than for large companies, as the smaller the company is, the more difficult legislation compliance appear [3]. The relevance for SMEs of factors in the governmental category may also lie in the role of regulation in inducing compliance [68]. SMEs might perceive governmental initiatives as a catalyst for complying and, thus, adopting sustainable manufacturing practices. This observation is linked with previous findings on the reactive approach SMEs often have toward environmental regulation, acting only when there is a specific requirement to do so, and otherwise focusing on daily business operations [19].

Barriers and enablers for the adoption of sustainable manufacturing rarely exist in isolation. More often, they are interconnected with other barriers and enablers [34]. The barrier of using outdated technology (B56), for example, is expected to be exacerbated by other barriers, such as lack of government subsidies (B33), lack of financial resources (B43), and difficulties in accessing financial capital (B44). We believe that these additional barriers make the first barrier more difficult to mitigate. Increased availability of government subsidies increases the probability that SMEs will purchase cleaner technologies [69]. Another example of the interconnection between barriers was pointed out by Oliveira Neto et al. [24], who reported that financial barriers, and more specifically, the lack of financial resources, have a significant impact on technological and informational barriers (e.g., low skilled labor (B59); use of outdated technology (B56); inadequate development of worker's new skills (B60), etc.). Likewise, the barrier resistance to change (B18) may be linked with the barrier lack of trust of SMEs' owners (B3), which makes SMEs' owners resistant to share sharing information about their companies. As a result, SMEs owners become reluctant to implement changes or acknowledge that changes are required, resulting in inertia [70].

The evident preponderance of barriers, over enablers, for sustainable manufacturing in SMEs may be rooted in the inherent characteristics of SMEs, which include limited human and financial resources, problems with accessing and managing knowledge, focus on daily operations, short-term priorities, and little focus on management practices [48,49]. These characteristics may cause SMEs to more frequently encounter difficulties meeting environmental and social standards than larger companies. The preponderance of barriers over enablers might be also affected by the infancy stage of research on sustainable operations management in SMEs (large enterprises being the main subjects of research in this area), resulting in limited external knowledge for implementing sustainable manufacturing practices in SMEs. As a result, guidelines or benchmarks for the adoption of sustainable manufacturing in SMEs are still lacking [49] and highly needed [25,71]. Another way in which the inherent characteristics of SMEs may influence enablers and barriers is exemplified by the fact that the commonly mentioned enabler is knowledge and social networks (E6). This enabler seems to be vital for SMEs as these organizations are commonly found to lack technical knowledge, face difficulties accessing technical information, and do not have all the necessary competence in-house. 
Flexibility and risk-taking have been described as common characteristics of SMEs [48,72]. Nevertheless, when it comes to the adoption of sustainable manufacturing practices, researchers have found that SMEs experience resistance to change (B18) while adopting these practices. This resistance may be a result of the typical short-term sight of the SMEs, where planning is often limited to the internal operational levels where performance is measured [73]. Although comprehensive methods have been proposed to evaluate sustainable manufacturing performance at the product and process levels, holistic approaches to evaluate sustainable manufacturing performance at systems level are still lacking [74]. Similarly, Mittal and Sangwan, [75], observed that SMEs, particularly in emerging and developing countries, show resistance to change, even to the adoption of better manufacturing and management systems.

It was found that some of the barriers and enablers could not be classified as exclusively enablers or exclusively barriers because they were situational and were indicated as 'factors' by the original authors. Two examples of these factors refer to public pressure and energy prices, in our study identified as: weak public awareness and pressure on SMEs (B52), pressure from markets and clients (E24), low energy prices (B54), and high energy prices (E217). These can be considered enablers by SMEs in developed economies within countries with highly responsive environmental and social policies and high levels of public awareness. At the same time, these two factors would be considered barriers for SMEs in other countries where sustainability awareness and policies are not prioritized or aligned, as is commonly the case in developing economies.

Furthermore, some of the identified barriers and enablers for SMEs tend to vary according to the level of development of the country in which the SMEs being studied were located. Fernandez-Viñé et al. [36], for example, observed that European SMEs considered the lack of infrastructure and technology to be a barrier, while Venezuelan SMEs recognized the insensitivity of buyers and end users as a barrier. Similarly, some of the studies conducted in developing countries in the Caribbean [38] or in China [22] reported the lack of a market for sustainable sound products as a clear barrier, while other studies viewed market demand for green products as an enabler of sustainable manufacturing [69]. Nevertheless, similarities in the barriers affecting SMEs in developed and developing countries have been also reported in the literature. Jabbour and Puppim-De-Oliveira [71] carried out a study between SMEs clusters in Japan and Brazil, concluding that both clusters were affected by similar barriers: lack of information and knowledge on sustainability, and competition with less-expensive products manufactured without social and environmental considerations.

Our study identified substantially more barriers (61) than enablers (35). This result contrasts earlier research, such as Walker at al. [35], where more enablers than barriers to environmental supply chain management were found. Our study also differs from the findings in Govindan and Bouzon [44], which found a similar number of barriers (36) and enablers (37) for reverse logistics, and of Bhanot et al. [25], that observed similar numbers of barriers (29) and enablers (22) for sustainable manufacturing in both large and SMEs. Given that our study focused exclusively on SMEs, the sharp difference in the numbers of barriers (61) and enablers (35) we identified may be the result of inherent characteristics of SMEs.

Regarding the origin of the barriers and enablers, our study did not identify any marked differences between the internal (51\%) and external barriers (49\%), and the internal $(49 \%)$ and external (51\%) enablers faced by SMEs. This even distribution of internal and external barriers and enablers contrasts with a review by Walker et al. [35], who found a predominance of internal barriers over external barriers to environmental supply chain management within large companies. However, after carrying out an empirical study, Walker et al. [35] found a similar distribution of internal and external barriers.

Our study suggests that the barriers and enablers to sustainable manufacturing within SMEs have similarities with the ones experienced by both large enterprises and SMEs. Mittal et al. [76] encountered similar enablers and barriers to green manufacturing im- 
plementation (disregarding organization size). Similar enablers identified were: strict legislation, incentives, public and peer pressure, cost savings, supply chain pressure, top management commitment, public image, and skilled staff. Common barriers included: weak legislation and low enforcement; high implementation costs; low managerial priority toward sustainable manufacturing; lack of managerial awareness; and limited access to sustainability literature. Nordin et al. [33] studied the barriers and enablers for sustainable manufacturing in both large enterprises and SMEs in Malaysia. They determined that environmental regulation was the most important enabler for environmental manufacturing practices, followed by top management commitment toward sustainable manufacturing practices and improved corporate image. The most significant barriers were: increases in the overall cost of implementation; lack of specific ideas of what to do and when to do it; and lack of awareness and understanding in companies of sustainability. Later, Bhanot et al. [25] studied the perceptions of barriers and enablers to sustainable manufacturing in both large enterprises and SMEs. Common barriers found in their study were: managers' negative preconceptions; resistance to change; lack of awareness of benefits from adopting sustainability; low availability of credit; high investment costs; and low returns of environmentally sustainable technologies. Common enablers were: government-provided information; education and training systems; managerial support; knowledge networks; and reducing manufacturing costs. Some enablers pinpointed by Bhanot et al. [25] that are not present in our study were: the development of e-economy, improving quality, and attracting foreign investment. All barriers observed by Bhanot et al. [25] were also identified in our study. Recently, Miras-Rodríguez et al. [31] studied enablers for environmental manufacturing practices in both large enterprises and SMEs, of which the following were also identified in our study: engaged top management to environmental responsibility; employee values aligned with sustainability; increased awareness of environmental issues among customers; and current government legislation. Enablers observed by Miras-Rodríguez et al. [31] that were not found in our study were: championing efforts by individual employees or small groups of employees; employee problem-solving teams; the threat of future government legislation; and the belief that it is possible to reduce costs while protecting the environment.

In summary, the results from our study suggest that, although certain similarities exist in the barriers and enablers affecting SMEs and large enterprises, further research comparing these bodies of studies is needed. We believe the impact of similar barriers may be more acute for SMEs than for large enterprises because of the lack of resources in SMEs.

\subsection{Critical Enablers Addressing Identified Barriers}

It was found that the most critical enablers, i.e., the enablers expected to mitigate the highest number of identified barriers (see Figure 5), in descending order of importance, were: knowledge networks and social networks (E6); increased support from large sized customers (E25); external cooperation (E26); provide government sponsored platforms supporting SMEs (E15); environmental management systems (EMAS and ISO 14001) (E11); government provided information on sustainable manufacturing (E16); managerial support toward sustainability (E1); education and training systems to improve operations (E35); and pressure from supply chains (E28). Knowledge and social networks (E6) constituted the top critical enabler expected to mitigate the highest number of barriers ( 22 barriers), confirming earlier findings in the literature that SMEs benefit from the professional advice received from academic collaborations [77]. Similarly, Triguero et al. [69] stated that collaborative networks between SMEs and research institutions are essential for the development of cleaner technologies by SMEs. Spence and Schmidpeter [78] accentuated the importance of SMEs' contributions to the development of social capital, which will result in improved social performance of the SME. In terms of the economic growth of SMEs, Shah et al. [79] noted the importance of social networking as a tool that enables SMEs to cooperate and collaborate with other firms in order to access and use scarce resources or resources that are not available to these SMEs. Social networks are critical as they constitute the social capital of SMEs and, thus, are crucial to a company's competitiveness and longevity. The 
European Commission also highlighted the importance of SME networks, and SME-specific associations at the European or national level, in supporting the sustainability progress of SMEs [3]. Organizations and associations representing SMEs are expected to facilitate the transfer of knowledge accumulated by third parties by: working in collaborative projects with several SMEs centered on solutions to the main environmental issues in the sector causing increased costs; and by providing sector specific guidelines for improving environmental performance.

Six out of the nine critical enablers are external, which highlights the crucial role that external parties (e.g., Government, large-customers) have in helping SMEs improve their sustainability performance. That said, it is evident that most of the identified barriers to sustainable manufacturing could be addressed through knowledge and education provided by either government or large customers; knowledge networks and social networks; or external cooperation.

Our analysis showed that enablers belonging to the OMA category had the potential to contribute the most toward addressing or overcoming the identified barriers to sustainable manufacturing. Table 3, Figures 4 and 5, and Appendix A Figure A3 reflect this by showing that OMA enablers most often contributed partially or directly to the mitigation of barriers. This result suggests that SMEs will have greater potential to mitigate barriers to sustainable manufacturing, by focusing on the enablers related to the OMA category. Moreover, this study found that OMA enablers could mitigate barriers in all categories. Conversely, financial enablers were only able to mitigate barriers related to financial aspects. This result may contradict the general belief that financing alternatives are the solution to many types of barriers, although it is consistent with results by Hillary [21] which found that the lack of human resources, not financial ones, in micro-sized and small enterprises were more decisive when introducing and maintaining environmental practices and systems. The importance of having appropriate human resources might be related to why low skilled labor constitutes one of the most commonly mentioned barriers in our overview.

\subsection{Limitations}

Prior work suggests that industrial barriers for SMEs vary from one industry sector to another, so the industrial barriers faced by one SME may be different from those faced by another operating in a different sector [34,80]. Hence, this study did not intend to present industrial barriers but, instead, to present an outlook of barriers and enablers faced by manufacturing SMEs adopting sustainable manufacturing.

A number of papers in the final sample used as theoretical foundation and thus, were strongly influenced by two pivotal studies: Hillary [21] and Cagno et al. [28]. Hillary's seminal work on barriers and enablers for EMS within SMEs influenced many other studies in our final sample of papers e.g., [71,81]. Similarly, Cagno et al. [28] presented a set of barriers to the adoption of energy-saving practices in SMEs again influencing other studies in our final sample $[37,39,82]$. The influence of these two papers could, therefore, have an impact on the factors we identified and, hence, the results of the present study.

Several papers specifically addressed barriers and enablers for the adoption of energyefficient practices by SMEs. This might be as environmentally sustainable practices being strongly associated to cost-cutting strategies, being the main motivation to reduce cost found to be the main driver to energy efficiency in SMEs [56]. Moreover, saving energy is considered among one the most common resource efficiency actions being undertaken by SMEs, alongside minimizing waste and saving materials [5]. Another aspect that may have contributed to this number of papers addressing energy efficiency practices, hence affecting our results, might be the presence in the final sample of a group of authors publishing and co-authoring papers on energy efficiency in SMEs [37,39,43,82].

It is worthy of note that some factors could be classified in more than one category. Similarly, it is relevant to acknowledge that there is no universally agreed classification system for barriers and enablers for sustainable manufacturing and that any system for categorizing enablers and barriers is, to some extent, arbitrary. Nevertheless, the expectation 
is that our categorization will facilitate explicit and systematic synthesis and interpretation of the existing body of evidence on barriers and enablers for adopting sustainable manufacturing in SMEs.

\section{Conclusions and Further Research}

This paper fills the gaps in current literature regarding the identification of barriers and enablers faced by manufacturing SMEs related to the adoption of sustainable manufacturing practices. It also describes what enablers can be used to mitigate the identified barriers for the adoption of sustainable manufacturing.

This paper proposed a categorization of the barriers and enablers for the adoption of sustainable manufacturing by SMEs, based on a systematic review of the existing literature on the topic. Unlike most studies addressing these factors, our study not only presented a categorization but also proposed corresponding descriptions and suggested what enablers might mitigate the identified barriers. This study confirmed that researchers continue to use a wide range of terminology in the literature when referring to barriers and enablers. Discrepancies are reflected in the terms enabler and drivers being used interchangeably in the literature.

In total, seven categories for classifying the barriers and enablers for the adoption of sustainable manufacturing within SMEs were identified: OMA; informational; governmental; financial; training and skills development; market and business context; and technological. Despite that our proposed set of categories was found to be similar to previous categorizations, it differs from them in that they considered the triple bottom line approach, described the categories and factors, and included the categories training and skills development, as well as informational.

The systematic literature review carried out in this study found that the barriers most frequently mentioned in the literature faced by SMEs while adopting sustainable manufacturing belong to the OMA, training and skills development, informational and financial categories (see Figure A1). It was found that most of the barriers in the literature were related to specific sustainable manufacturing practices, compared to a significantly smaller number of barriers toward more sustainability integrated approaches. This study also found that the technology category presented only barriers to sustainable manufacturing in SMEs. This finding strongly suggests technology suppliers should increase the availability of technologies for sustainable manufacturing that are suitable for SMEs.

The enablers most often mentioned in the literature are equally distributed between the categories OMA, financial, market and business context, and government (see Figure A2). Moreover, this study concluded that there are critical enablers with the potential to mitigate a considerable number of barriers, such as: knowledge networks and social networks (E6), increased support from large sized customers (E25), external cooperation (E26), provide government-sponsored platforms supporting SMEs (E15), environmental management systems (E11), government provided information on sustainable manufacturing (E16), managerial support toward sustainability (E1), education and training systems to improve operations (E35), and pressure from supply chains (E28). Knowledge and social networks seem to be an outstanding enabler expected to mitigate 22 out of 61 barriers. Many of the identified barriers to sustainable manufacturing could be mitigated by participating in knowledge networks and social networks; providing knowledge and education through government or large customers; or by external cooperation with other stakeholders.

The OMA category contained the most enablers expected to make the greatest contribution to mitigating the identified barriers, followed by the market and business context and governmental categories. Thus, SMEs aiming to adopt sustainable manufacturing practices or improve their sustainability performance are encouraged to focus on enablers in these three categories.

Most of the identified barriers are of external origin; it can be stated that academics, technology providers, government, industrial associations, financial institutions, respec- 
tively, are expected to increase the support and offers provided to SMEs regarding ways to mitigate the barriers.

Special attention needs to be given to the following barriers, which had no mitigating enablers: difficulty quantifying the financial performance of environmental investments (B48), traditional financial accounting systems excluding environmental costs (B49); frequent changes in environmental regulations (B39); absence of single authoritative body interpreting EMSs (B40); and corruption (B41). Policy-makers and key stakeholders are called on to allocate additional effort and resources to help mitigate these barriers.

The results reported in this study have both theoretical and practical implications. The theoretical contribution is the identification and categorization of barriers and enablers faced by manufacturing SMEs considering the triple bottom line approach. The practical contribution of this study was to obtain insights about of barriers and enablers and the critical enablers that could assist in mitigating barriers, which will be valuable to manufacturing SMEs interested in adopting sustainable manufacturing practices or improving sustainability performance. This study also constitutes a valuable source of knowledge for policy-makers that may help them make informed decisions and design policies that address the specific needs of SMEs.

Future research could proceed in the following directions. Firstly, it could explore the perceptions of SME stakeholders (e.g., large customers and government) of the barriers and enablers identified. Secondly, it could investigate the perceived importance of the identified barriers and enablers from a managerial perspective, to determine whether the literature reflects the current perception of SMEs toward these factors. In addition, finally, it could empirically validate the identified barriers and enablers within manufacturing SMEs in different industrial sectors and in different areas of the world.

Author Contributions: Conceptualization, C.L.A., and K.S.; Formal analysis, C.L.A.; Investigation, C.L.A.; Methodology, C.L.A., and K.S.; Supervision, K.S. and G.J.; Visualization, C.L.A., and K.S.; Writing—original draft, C.L.A.; Writing—review \& editing, K.S., and G.J. All authors have read and agreed to the published version of the manuscript.

Funding: This research received no external funding.

Institutional Review Board Statement: Not applicable.

Informed Consent Statement: Not applicable.

Data Availability Statement: Not applicable.

Acknowledgments: We gratefully acknowledge the anonymous reviewers for their time, insightful suggestions and constructive comments provided to this manuscript, and School of Engineering, Jönköping University, Sweden, who enabled this research.

Conflicts of Interest: The authors declare no conflict of interest. 


\section{Appendix A}

Table A1. Overview of barriers and enablers for the adoption of sustainable manufacturing by manufactuing SMEs.

\section{Barriers to Sustainable Manufacturing Adoption by Manufacturing SMEs}

\begin{tabular}{|c|c|c|c|c|c|}
\hline Cat. & Subcategory & Origin & Description & References & Freq \\
\hline \multirow{12}{*}{ 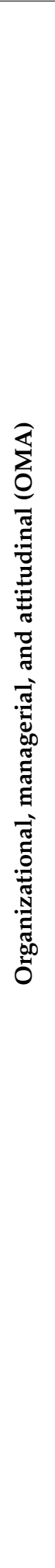 } & $\begin{array}{l}\text { B1. Manager's lack of } \\
\text { awareness about the company's } \\
\text { environmental impact }\end{array}$ & I & $\begin{array}{l}\text { Managers lack knowledge, } \\
\text { understanding and awareness of } \\
\text { environmental issues. }\end{array}$ & {$[21,23,64,70,71,81,83-85]$} & 9 \\
\hline & $\begin{array}{l}\text { B2. Manager's } \\
\text { misunderstandings and } \\
\text { pessimistic preconceptions } \\
\text { about sustainable } \\
\text { manufacturing }\end{array}$ & I & $\begin{array}{l}\text { Pessimistic preconceptions of } \\
\text { unmanageable time, and high-cost } \\
\text { requirements to adopt sustainable } \\
\text { manufacturing practices (e.g., costs, } \\
\text { lack of commercial viability, lack of } \\
\text { profitability, technical challenges). }\end{array}$ & $\begin{array}{l}{[21,37,38,50,55,56,71,77,81} \\
83,84,86-88]\end{array}$ & 14 \\
\hline & B3. Lack of trust of SME owners & I & $\begin{array}{l}\text { SMEs' owners' resistance to share } \\
\text { information about their companies. }\end{array}$ & {$[70,85]$} & 2 \\
\hline & $\begin{array}{l}\text { B4. Expectations of excessively } \\
\text { high profitability from energy } \\
\text { efficiency practices }\end{array}$ & I & $\begin{array}{l}\text { Managers' expectations of excessively } \\
\text { high profitability from energy } \\
\text { efficiency practices. }\end{array}$ & [56] & 1 \\
\hline & $\begin{array}{l}\text { B5. Incompatibility of owner's } \\
\text { ethics and personal values with } \\
\text { sustainability }\end{array}$ & I & $\begin{array}{l}\text { Lack of interest in sustainable } \\
\text { manufacturing practices from SMEs' } \\
\text { owners. }\end{array}$ & {$[21,37,81,84,85,88]$} & 6 \\
\hline & $\begin{array}{l}\text { B6. Organizational culture not } \\
\text { aligned with sustainability } \\
\text { values }\end{array}$ & I & $\begin{array}{l}\text { Underdeveloped culture involving } \\
\text { sustainability values or principles. }\end{array}$ & {$[21,81,83,85,88]$} & 5 \\
\hline & $\begin{array}{l}\text { B7. Low managerial priority } \\
\text { toward undertaken sustainable } \\
\text { manufacturing practices }\end{array}$ & I & $\begin{array}{l}\text { Higher priority given to production } \\
\text { expansion or increasing market share } \\
\text { over allocation of resources to } \\
\text { sustainable technologies. }\end{array}$ & {$[22-24,36,37,83,85-87]$} & 9 \\
\hline & $\begin{array}{l}\text { B8. Lack of managerial } \\
\text { competence in } \\
\text { owners/managers of SMEs }\end{array}$ & I & $\begin{array}{l}\text { Managers/owners of SMEs lack the } \\
\text { basic managerial and technical } \\
\text { capacity to implement sustainable } \\
\text { manufacturing practices and to make } \\
\text { of their enterprise a learning } \\
\text { organization. }\end{array}$ & {$[22,24,77,79,86]$} & 5 \\
\hline & $\begin{array}{l}\text { B9. Lack of time for planning, } \\
\text { execution and review of } \\
\text { sustainable manufacturing } \\
\text { practices }\end{array}$ & I & $\begin{array}{l}\text { Lack of time of SMEs' } \\
\text { owners/managers and staff to engage } \\
\text { in sustainability-related activities. }\end{array}$ & $\begin{array}{l}{[21,23,24,37,39,65,78,79,81,} \\
82,85-87,89]\end{array}$ & 14 \\
\hline & $\begin{array}{l}\text { B10. Perceived conflicts } \\
\text { between environmental } \\
\text { practices and other business } \\
\text { objectives }\end{array}$ & I & $\begin{array}{l}\text { Perceived trade-offs between } \\
\text { environmental practices with other } \\
\text { manufacturing performance } \\
\text { objectives (e.g., quality, cost, delivery) } \\
\text { regarding resources, energy usage, } \\
\text { materials, and waste emissions. }\end{array}$ & {$[36,37,83,87]$} & 4 \\
\hline & $\begin{array}{l}\text { B11. Concerns that } \\
\text { environmental investments } \\
\text { undermine competitiveness }\end{array}$ & I & $\begin{array}{l}\text { Additional costs from adopting } \\
\text { environmental technologies might } \\
\text { undermine the company's } \\
\text { competitiveness in the marketplace. }\end{array}$ & [22] & 1 \\
\hline & B12. Lack of human resources & I & $\begin{array}{l}\text { Limited personnel occupied with } \\
\text { daily tasks and working alone on } \\
\text { identification and implementation of } \\
\text { sustainable manufacturing practices. }\end{array}$ & {$[23,65,77,79,83,85,86]$} & 7 \\
\hline
\end{tabular}


Table A1. Cont.

Barriers to Sustainable Manufacturing Adoption by Manufacturing SMEs

\begin{tabular}{|c|c|c|c|c|c|}
\hline Cat. & Subcategory & Origin & Description & References & Freq \\
\hline \multirow{10}{*}{ 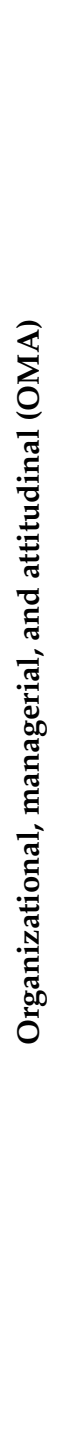 } & B13. Multifunctional staff & I & $\begin{array}{l}\text { Multifunctional staff often associated } \\
\text { with interruptions in the adoption of } \\
\text { sustainable manufacturing practices. }\end{array}$ & {$[21,81,86]$} & 3 \\
\hline & $\begin{array}{l}\text { B14. Low involvement of } \\
\text { workers }\end{array}$ & I & $\begin{array}{l}\text { Workers lack interest in sustainable } \\
\text { manufacturing practices; thus, their } \\
\text { involvement in such practices is low. } \\
\text { Lack of accepting suggestions from } \\
\text { employees. }\end{array}$ & {$[24,79,89]$} & 3 \\
\hline & $\begin{array}{l}\text { B15. Difficulties attracting and } \\
\text { retaining workers }\end{array}$ & I & $\begin{array}{l}\text { SMEs face a high turnover of staff } \\
\text { and difficulties attracting new } \\
\text { workers. }\end{array}$ & {$[21,77,81]$} & 3 \\
\hline & $\begin{array}{l}\text { B16. Lack of awareness of the } \\
\text { benefits derived from } \\
\text { sustainable manufacturing } \\
\text { practices }\end{array}$ & I & $\begin{array}{l}\text { Staff and managers lack interest and } \\
\text { awareness of the economic and } \\
\text { environmental benefits of sustainable } \\
\text { manufacturing practices. }\end{array}$ & $\begin{array}{l}{[21,23,37-} \\
39,56,70,71,77,81-85,89]\end{array}$ & 15 \\
\hline & B17. Risk perception & I & $\begin{array}{l}\text { Overestimation of the investment } \\
\text { risks, resulting from poor knowledge } \\
\text { of energy efficiency technology. }\end{array}$ & {$[39,56,82,85]$} & 4 \\
\hline & B18. Resistance to change & I & $\begin{array}{l}\text { Management fears the risks of } \\
\text { modifying current production } \\
\text { processes and technologies and/or } \\
\text { replacing them with new ones. Staff } \\
\text { resistant to change to new practices. }\end{array}$ & $\begin{array}{l}{[21-24,37-} \\
39,55,77,81,83,87,89]\end{array}$ & 13 \\
\hline & $\begin{array}{l}\text { B19. Lack of internal marketing } \\
\text { of practices and systems }\end{array}$ & I & $\begin{array}{l}\text { Lack of internal marketing of } \\
\text { practices and systems that promote } \\
\text { among employees the sustainable } \\
\text { objectives. }\end{array}$ & {$[21,81]$} & 2 \\
\hline & B20. Lack of communication & I & $\begin{array}{l}\text { Lack of communication vertically and } \\
\text { horizontally in SMEs. }\end{array}$ & {$[55,79,81,89]$} & 4 \\
\hline & $\begin{array}{l}\text { B21. Lack of continuity in the } \\
\text { adoption of sustainable } \\
\text { manufacturing practices }\end{array}$ & I & $\begin{array}{l}\text { Lack of a systemic approach toward } \\
\text { the implementation of sustainable } \\
\text { manufacturing. }\end{array}$ & {$[21,23,55,81]$} & 4 \\
\hline & $\begin{array}{l}\text { B22. Lack of enough } \\
\text { environmental progress }\end{array}$ & I & $\begin{array}{l}\text { Current environmental progress does } \\
\text { not motivate the improvement. }\end{array}$ & {$[21,81]$} & 2 \\
\hline \multirow{3}{*}{ 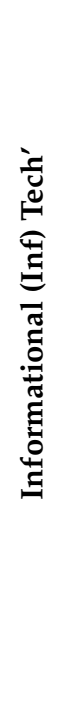 } & $\begin{array}{l}\text { B23. Lack of access to external } \\
\text { technical knowledge to adopt } \\
\text { sustainable manufacturing } \\
\text { practices }\end{array}$ & $\mathrm{E}$ & $\begin{array}{l}\text { Insufficient offerings of external } \\
\text { technical support to SMEs, lack of } \\
\text { access to technical information, lack } \\
\text { of available external knowledge for } \\
\text { implementing sustainable } \\
\text { manufacturing. Lack of metrics, } \\
\text { benchmarks, and guidance for } \\
\text { adopting sustainable manufacturing. }\end{array}$ & $\begin{array}{l}{[21,22,24,37,55,56,65,70,71,} \\
77,81,83-85,87-90]\end{array}$ & 18 \\
\hline & $\begin{array}{l}\text { B24. Little knowledge on } \\
\text { sustainability in SMEs }\end{array}$ & I & $\begin{array}{l}\text { A lack of knowledge of sustainability } \\
\text { concepts in SMEs. This due to the } \\
\text { lack of, or limited access to, } \\
\text { sustainability-related literature } \\
\text { focused on SMEs. }\end{array}$ & $\begin{array}{l}{[21-24,38,70,71,77,81,85} \\
86,89,90]\end{array}$ & 13 \\
\hline & $\begin{array}{l}\text { B25. Lack of knowledge on } \\
\text { environmental management } \\
\text { systems and difficulty for } \\
\text { certification }\end{array}$ & I & $\begin{array}{l}\text { SMEs are poorly informed about } \\
\text { EMSs (EMASs and ISO 14001) and its } \\
\text { benefits. } \\
\text { High cost of certification/verification } \\
\text { of EMSs. }\end{array}$ & {$[21,81]$} & 2 \\
\hline
\end{tabular}


Table A1. Cont.

Barriers to Sustainable Manufacturing Adoption by Manufacturing SMEs

\begin{tabular}{|c|c|c|c|c|c|}
\hline Cat. & Subcategory & Origin & Description & References & Freq \\
\hline \multirow{7}{*}{ 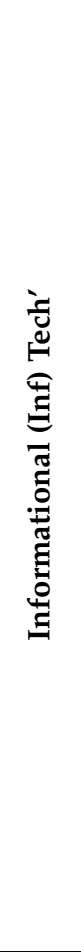 } & $\begin{array}{l}\text { B26. Lack of awareness } \\
\text { programs conducted locally }\end{array}$ & $\mathrm{E}$ & $\begin{array}{l}\text { Lack of awareness programs about } \\
\text { the importance of adopting } \\
\text { sustainable manufacturing. }\end{array}$ & {$[23,37,65,77,89]$} & 5 \\
\hline & $\begin{array}{l}\text { B27. Lack of access to } \\
\text { information on specific } \\
\text { technologies }\end{array}$ & $\mathrm{E}$ & $\begin{array}{l}\text { Information regarding sustainable } \\
\text { technologies is not available to SMEs' } \\
\text { managers. This affects the perceived } \\
\text { managerial complexity of new } \\
\text { technology. }\end{array}$ & {$[56,86,89]$} & 3 \\
\hline & $\begin{array}{l}\text { B28. Information from } \\
\text { technology suppliers is not clear } \\
\text { for SMEs }\end{array}$ & $\mathrm{E}$ & $\begin{array}{l}\text { Lack of information on costs and } \\
\text { benefits of energy efficient } \\
\text { technologies }\end{array}$ & {$[39,82]$} & 2 \\
\hline & $\begin{array}{l}\text { B29. Lack of information on real } \\
\text { costs and benefits of energy } \\
\text { efficient technologies }\end{array}$ & $\mathrm{E}$ & $\begin{array}{l}\text { Lack of information and little } \\
\text { knowledge of costs and benefits of } \\
\text { energy efficient technologies. }\end{array}$ & {$[39,82,85]$} & 3 \\
\hline & $\begin{array}{l}\text { B30. Lack of data on energy } \\
\text { consumption }\end{array}$ & $\mathrm{I}$ & $\begin{array}{l}\text { Lack of data on energy consumption } \\
\text { patterns and efficiency measures is } \\
\text { common among SMEs. }\end{array}$ & {$[23,56]$} & 2 \\
\hline & $\begin{array}{l}\text { B31. Low trustworthiness of the } \\
\text { information source }\end{array}$ & $\mathrm{E}$ & $\begin{array}{l}\text { Quality of the available information } \\
\text { on energy efficiency technologies. }\end{array}$ & {$[23,39,56,82]$} & 4 \\
\hline & $\begin{array}{l}\text { B32. Lack of information on } \\
\text { economic incentives }\end{array}$ & $\mathrm{E}$ & $\begin{array}{l}\text { Information regarding economic } \\
\text { incentives is not available to } \\
\text { managers. }\end{array}$ & {$[24,56]$} & 2 \\
\hline \multirow{8}{*}{ 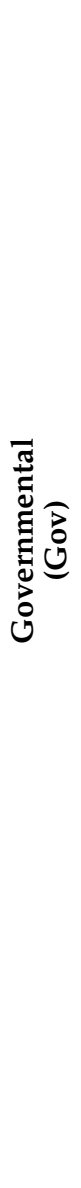 } & $\begin{array}{l}\text { B33. Lack of governmental } \\
\text { incentive policies; subsidies, } \\
\text { taxes. }\end{array}$ & $\mathrm{E}$ & $\begin{array}{l}\text { Lack of governmental subsidies and } \\
\text { other types of incentives. Lack of } \\
\text { access to information on economic } \\
\text { incentives. }\end{array}$ & {$[22-24,69,70,77,84,89]$} & 8 \\
\hline & $\begin{array}{l}\text { B34. Lack of incentives for local } \\
\text { collaborative networks and } \\
\text { associations }\end{array}$ & $\mathrm{E}$ & $\begin{array}{l}\text { Lack of incentives for SMEs to build } \\
\text { local collaborative networks. }\end{array}$ & {$[21,69,77,78,81]$} & 5 \\
\hline & $\begin{array}{l}\text { B35. Certain subsidies that } \\
\text { impede sustainable } \\
\text { manufacturing }\end{array}$ & $\mathrm{E}$ & $\begin{array}{l}\text { Economic subsidies for business } \\
\text { resource inputs may be a significant } \\
\text { disincentive to environmental } \\
\text { sustainability. }\end{array}$ & [55] & 1 \\
\hline & $\begin{array}{l}\text { B36. Lack of local guidance for } \\
\text { adopting sustainable } \\
\text { manufacturing }\end{array}$ & $\mathrm{E}$ & $\begin{array}{l}\text { Lack of local guidance for adopting } \\
\text { sustainable manufacturing/CSR. }\end{array}$ & {$[24,77,84,88-90]$} & 6 \\
\hline & $\begin{array}{l}\text { B37. Lack of effective legislation } \\
\text { and a weak regulatory } \\
\text { environment }\end{array}$ & $\mathrm{E}$ & $\begin{array}{l}\text { Lenient environmental legislation } \\
\text { and weakly enforced environmental } \\
\text { legislation applicable to SMEs. }\end{array}$ & $\begin{array}{l}{[21,22,24,55,65,69,77,81,83,} \\
84,89,90]\end{array}$ & 12 \\
\hline & $\begin{array}{l}\text { B38. Difficulty complying with } \\
\text { regulations }\end{array}$ & $\mathrm{E}$ & $\begin{array}{l}\text { SMEs find it difficult to comply with } \\
\text { regulations. SMEs perceive } \\
\text { regulations as complex and } \\
\text { challenging to understand. }\end{array}$ & {$[21,23,24,36,55,77,86]$} & 7 \\
\hline & $\begin{array}{l}\text { B39. Frequent changes in } \\
\text { environmental regulations }\end{array}$ & $\mathrm{E}$ & $\begin{array}{l}\text { Frequent changes in environmental } \\
\text { regulations and lack of resources to } \\
\text { track down the incoming } \\
\text { environmental legislation. }\end{array}$ & {$[24,50,81]$} & 3 \\
\hline & $\begin{array}{l}\text { B40. Absence of a single } \\
\text { authoritative body to interpret } \\
\text { EMSs }\end{array}$ & $\mathrm{E}$ & $\begin{array}{l}\text { Absence of a single authoritative } \\
\text { body to interpret EMSs. }\end{array}$ & {$[21,81]$} & 2 \\
\hline
\end{tabular}


Table A1. Cont.

Barriers to Sustainable Manufacturing Adoption by Manufacturing SMEs

\begin{tabular}{|c|c|c|c|c|c|}
\hline Cat. & Subcategory & Origin & Description & References & Freq \\
\hline \multirow{2}{*}{ 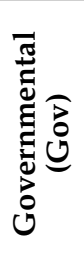 } & B41. Corruption & $\mathrm{E}$ & $\begin{array}{l}\text { Highly corrupt regulatory authorities } \\
\text { and officials mostly in developing } \\
\text { countries. } \\
\text { Unfair competition due to corruption. }\end{array}$ & {$[79,84]$} & 2 \\
\hline & $\begin{array}{l}\text { B42. Low political participation } \\
\text { of SMEs }\end{array}$ & $\mathrm{E}$ & $\begin{array}{l}\text { SMEs have small or limited political } \\
\text { participation. }\end{array}$ & [78] & 1 \\
\hline \multirow{7}{*}{ 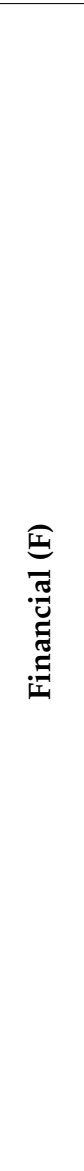 } & B43. Lack of financial resources & I & Low availability of capital in SMEs. & $\begin{array}{l}{[21,23,24,37,39,50,55,65,70,} \\
81-83,85-87]\end{array}$ & 15 \\
\hline & $\begin{array}{l}\text { B44. Difficulty accessing } \\
\text { financial capital }\end{array}$ & $\mathrm{E}$ & $\begin{array}{l}\text { Limited financing channels designed } \\
\text { or available for SMEs. Lack of funds } \\
\text { for environmentally related projects } \\
\text { (e.g., capital devoted to energy } \\
\text { efficiency investments). }\end{array}$ & $\begin{array}{l}{[21-24,50,56,70,77,79,81,} \\
85,86,90]\end{array}$ & 13 \\
\hline & $\begin{array}{l}\text { B45. High investment costs and } \\
\text { low returns of environmentally } \\
\text { sustainable technologies }\end{array}$ & $\mathrm{E}$ & $\begin{array}{l}\text { The initial capital costs of adopting } \\
\text { environmental technologies are often } \\
\text { higher than those of conventional } \\
\text { technologies. Low returns and longer } \\
\text { payback periods for environmentally } \\
\text { related investments. }\end{array}$ & {$[22-24,37-39,56,82,86,87]$} & 10 \\
\hline & $\begin{array}{l}\text { B46. Hidden costs of adopting } \\
\text { certain sustainable } \\
\text { manufacturing practices, and } \\
\text { compliance cost for regulations. }\end{array}$ & I E & $\begin{array}{l}\text { The aggregation of all the costs pre, } \\
\text { during, or post the adoption of } \\
\text { sustainable manufacturing practices } \\
\text { (e.g., research on technologies } \\
\text { available, disruption costs, training } \\
\text { personnel on using new technology). }\end{array}$ & {$[39,79,82]$} & 3 \\
\hline & B47. Cost of implementing CSR. & $\mathrm{E}$ & $\begin{array}{l}\text { Social compliance cost (time, other } \\
\text { types of costs) as a barrier to } \\
\text { implementing CSR. }\end{array}$ & {$[24,86,88]$} & 3 \\
\hline & $\begin{array}{l}\text { B48. Difficulty quantifying the } \\
\text { financial performance of } \\
\text { environmental investments }\end{array}$ & $\mathrm{E}$ & $\begin{array}{l}\text { Difficulty quantifying the financial } \\
\text { performance of environmental } \\
\text { investments. }\end{array}$ & [22] & 1 \\
\hline & $\begin{array}{l}\text { B49. Traditional financial } \\
\text { accounting systems exclude } \\
\text { environmental costs }\end{array}$ & $\mathrm{E}$ & $\begin{array}{l}\text { Traditional financial accounting } \\
\text { systems in SMEs fail to include } \\
\text { environmental costs and liabilities. }\end{array}$ & {$[55,77]$} & 2 \\
\hline \multirow{5}{*}{ 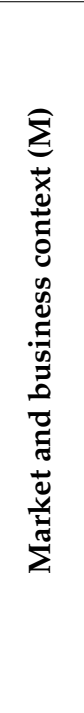 } & $\begin{array}{l}\text { B50. Lack of awareness of } \\
\text { international trends related to } \\
\text { environmental sustainability }\end{array}$ & $\mathrm{E}$ & $\begin{array}{l}\text { Lack of awareness regarding } \\
\text { international trends in legislation and } \\
\text { markets about the importance of } \\
\text { environmental aspects to business. }\end{array}$ & [83] & 1 \\
\hline & $\begin{array}{l}\text { B51. Competition with } \\
\text { less-expensive products }\end{array}$ & $\mathrm{E}$ & $\begin{array}{l}\text { Competition with markets and } \\
\text { less-expensive products excluding } \\
\text { environmental or social } \\
\text { considerations. }\end{array}$ & {$[71,83]$} & 2 \\
\hline & $\begin{array}{l}\text { B52. Weak public awareness } \\
\text { and pressure on SMEs }\end{array}$ & $\mathrm{E}$ & $\begin{array}{l}\text { Insufficient public pressure on SMEs } \\
\text { toward more benign sustainable } \\
\text { manufacturing practices. }\end{array}$ & {$[22,77]$} & 2 \\
\hline & $\begin{array}{l}\text { B53. Lack of awareness of SMEs } \\
\text { role on social capital }\end{array}$ & $\mathrm{E}$ & $\begin{array}{l}\text { Lack of awareness of media, } \\
\text { academia, and politicians of SMEs } \\
\text { adding to social capital. }\end{array}$ & [78] & 1 \\
\hline & B54. Low energy prices & $\mathrm{E}$ & $\begin{array}{l}\text { Low energy prices hindering } \\
\text { proactive actions to reduce energy } \\
\text { from SMEs. }\end{array}$ & {$[55,69]$} & 2 \\
\hline
\end{tabular}


Table A1. Cont.

Barriers to Sustainable Manufacturing Adoption by Manufacturing SMEs

\begin{tabular}{|c|c|c|c|c|c|}
\hline Cat. & Subcategory & Origin & Description & References & Freq \\
\hline \multirow{4}{*}{ 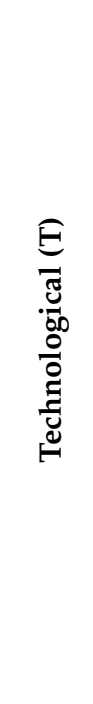 } & $\begin{array}{l}\text { B55. Need for additional } \\
\text { infrastructural modifications }\end{array}$ & I & $\begin{array}{l}\text { The adoption of new sustainable } \\
\text { manufacturing practices related to } \\
\text { infrastructure might result in } \\
\text { problems with space, infrastructure, } \\
\text { etc. }\end{array}$ & {$[22,23,38,85,89,90]$} & 6 \\
\hline & $\begin{array}{l}\text { B56. Use of outdated } \\
\text { technology }\end{array}$ & I & $\begin{array}{l}\text { Technologies used by SMEs are } \\
\text { outdated, and more sustainable and } \\
\text { modern alternatives (equipment and } \\
\text { substances) are unavailable. Lack of } \\
\text { alternative substances to substitute } \\
\text { the hazardous ones. }\end{array}$ & {$[24,37,55,77,89]$} & 5 \\
\hline & B57. Unavailable technology & $\mathrm{E}$ & $\begin{array}{l}\text { Technology suppliers not updated. } \\
\text { Cleaner technologies suitable for the } \\
\text { small-scale sector are limited. }\end{array}$ & {$[37,39,82,89]$} & 4 \\
\hline & $\begin{array}{l}\text { B58. Emphasis on end-of-pipe } \\
\text { process }\end{array}$ & I & $\begin{array}{l}\text { Emphasis on end-of-pipe process, as } \\
\text { opposed to making changes in the } \\
\text { processes. }\end{array}$ & {$[24,89]$} & 2 \\
\hline \multirow{3}{*}{ 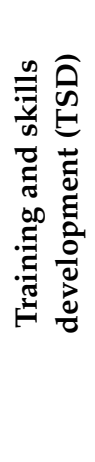 } & B59. Low skilled labour & I & $\begin{array}{l}\text { Limited in-plant expertise. Shortage } \\
\text { of skilled workers and core } \\
\text { competency. }\end{array}$ & $\begin{array}{l}{[21-24,36,56,65,70,77,79,} \\
81,83,85-87,90]\end{array}$ & 16 \\
\hline & $\begin{array}{l}\text { B60. Inadequate development } \\
\text { of workers' new skills, and lack } \\
\text { of technical training }\end{array}$ & I & $\begin{array}{l}\text { Inadequate development of workers' } \\
\text { new skills and capabilities. Lack of } \\
\text { training programs on developing, } \\
\text { performing and maintaining } \\
\text { sustainable manufacturing practices. }\end{array}$ & $\begin{array}{l}{[21,22,64,71,77,79,81,87,} \\
90]\end{array}$ & 9 \\
\hline & $\begin{array}{l}\text { B61. Lack of investment in } \\
\text { environmental education and } \\
\text { training }\end{array}$ & I & $\begin{array}{l}\text { SMEs often lack financial resources } \\
\text { for education and training on } \\
\text { environmental sustainability. }\end{array}$ & {$[21-23,70,81,86,90]$} & 7 \\
\hline \multirow{4}{*}{ 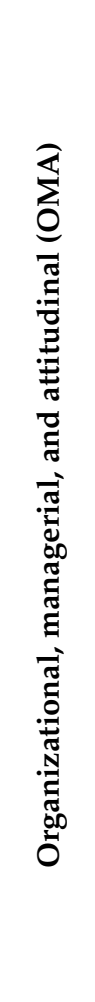 } & $\begin{array}{l}\text { E1. Managerial support and } \\
\text { effective leadership toward } \\
\text { sustainability }\end{array}$ & I & $\begin{array}{l}\text { Managerial support is pivotal to } \\
\text { initiate and maintain sustainable } \\
\text { manufacturing practices. } \\
\text { Inspiring managers or personnel } \\
\text { addressing sustainability issues could } \\
\text { convert sustainable manufacturing } \\
\text { adoption into an organizational } \\
\text { priority. }\end{array}$ & {$[23,37,39,43,64,85,90,91]$} & 8 \\
\hline & $\begin{array}{l}\text { E2. Managerial support toward } \\
\text { a collaborative environment for } \\
\text { innovation }\end{array}$ & I & $\begin{array}{l}\text { A favorable environment for } \\
\text { innovation by offering employees } \\
\text { training on innovation and creative } \\
\text { thinking; setting a reward system for } \\
\text { employees' innovation initiatives ... }\end{array}$ & [67] & 1 \\
\hline & E3. Long-term strategy & I & $\begin{array}{l}\text { With the establishment of a long-term } \\
\text { sustainability strategy, practices are } \\
\text { more likely to succeed, as will be } \\
\text { considered organizational priorities. }\end{array}$ & {$[37,39,43,85]$} & 4 \\
\hline & $\begin{array}{l}\text { E4. Realistic expectations about } \\
\text { benefits emerged from } \\
\text { sustainable manufacturing }\end{array}$ & I & $\begin{array}{l}\text { Increased awareness about the } \\
\text { expected benefits that may result } \\
\text { from adopting sustainable } \\
\text { manufacturing practices (e.g., cost } \\
\text { savings, new markets, positive } \\
\text { corporate image, improved } \\
\text { environmental and social } \\
\text { performance). }\end{array}$ & {$[37,39,43,65,91]$} & 5 \\
\hline
\end{tabular}


Table A1. Cont.

Barriers to Sustainable Manufacturing Adoption by Manufacturing SMEs

\begin{tabular}{|c|c|c|c|c|c|}
\hline Cat. & Subcategory & Origin & Description & References & Freq \\
\hline \multirow{10}{*}{ 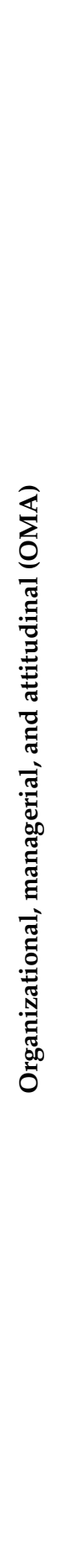 } & $\begin{array}{l}\text { E5. Develop continuously and } \\
\text { quick adaption to the } \\
\text { environment }\end{array}$ & I & $\begin{array}{l}\text { Continuous and quick adaptation is } \\
\text { an important competitive advantage } \\
\text { for companies. By continuously } \\
\text { improving SMEs expertise on } \\
\text { environmental practices, SMEs can } \\
\text { step ahead of their competition. }\end{array}$ & [65] & 1 \\
\hline & $\begin{array}{l}\text { E6. Knowledge networks and } \\
\text { social networks }\end{array}$ & $\mathrm{E}$ & $\begin{array}{l}\text { To participate, create and strengthen } \\
\text { social networks with financiers, } \\
\text { government bodies, suppliers' } \\
\text { associations, trade unions, } \\
\text { stakeholders. Sustainability } \\
\text { Partnership networks assist SMEs } \\
\text { with gaining knowledge, pooling } \\
\text { resources, and developing mutually } \\
\text { beneficial sustainable projects. }\end{array}$ & $\begin{array}{l}{[37,39,43,56,63,69,77-} \\
79,83,89]\end{array}$ & 11 \\
\hline & $\begin{array}{l}\text { E7. Interpersonal attitudes and } \\
\text { communications }\end{array}$ & I & $\begin{array}{l}\text { The presence of good interpersonal } \\
\text { attitudes and communications. }\end{array}$ & {$[56,64,85,89]$} & 4 \\
\hline & $\begin{array}{l}\text { E8. Adoption of lean } \\
\text { manufacturing and product } \\
\text { innovation strategies }\end{array}$ & I & $\begin{array}{l}\text { Lean product innovation strategies } \\
\text { enable SMEs to break into new } \\
\text { markets and to thereby become more } \\
\text { resilient to market changes. }\end{array}$ & {$[64]$} & 1 \\
\hline & $\begin{array}{l}\text { E9. Inclusiveness of workers } \\
\text { toward a more participatory } \\
\text { culture }\end{array}$ & I & $\begin{array}{l}\text { The acknowledgment that all } \\
\text { personnel within a company have } \\
\text { valuable contributions. Employees } \\
\text { share responsibility for sustainability } \\
\text { and a common concern for the } \\
\text { environment. }\end{array}$ & {$[37,39,43,64,90]$} & 5 \\
\hline & $\begin{array}{l}\text { E10. More efficient routines and } \\
\text { practices }\end{array}$ & I & $\begin{array}{l}\text { The problems with space and } \\
\text { infrastructure from sustainable } \\
\text { manufacturing practices might be } \\
\text { solved by developing more efficient } \\
\text { routines and practices. }\end{array}$ & [23] & 1 \\
\hline & $\begin{array}{l}\text { E11. Environmental } \\
\text { management systems (EMAS } \\
\text { and ISO 14001) }\end{array}$ & I & $\begin{array}{l}\text { SMEs having ISO } 14001 \text { certification } \\
\text { are likely to perform better on } \\
\text { environmental activities. }\end{array}$ & {$[37,39,43,65]$} & 4 \\
\hline & $\begin{array}{l}\text { E12. Use of business analysis } \\
\text { tools }\end{array}$ & I & $\begin{array}{l}\text { Business analysis process tools (i.e., } \\
\text { prioritization of environmental } \\
\text { aspects; stakeholder analysis; SWOT } \\
\text { analysis). }\end{array}$ & [83] & 1 \\
\hline & $\begin{array}{l}\text { E13. Availability of flexible and } \\
\text { innovative human resources }\end{array}$ & I & $\begin{array}{l}\text { To improve organizational and } \\
\text { sustainability performance, while } \\
\text { empowering personnel. }\end{array}$ & {$[64]$} & 1 \\
\hline & $\begin{array}{l}\text { E14. Flat management structure } \\
\text { and empowerment of the } \\
\text { workforce }\end{array}$ & I & $\begin{array}{l}\text { Application of a flat management } \\
\text { structure and the empowerment of } \\
\text { the workforce to self-organize. }\end{array}$ & {$[64]$} & 1 \\
\hline
\end{tabular}


Table A1. Cont.

Barriers to Sustainable Manufacturing Adoption by Manufacturing SMEs

\begin{tabular}{|c|c|c|c|c|c|}
\hline Cat. & Subcategory & Origin & Description & References & Freq \\
\hline \multirow{9}{*}{ 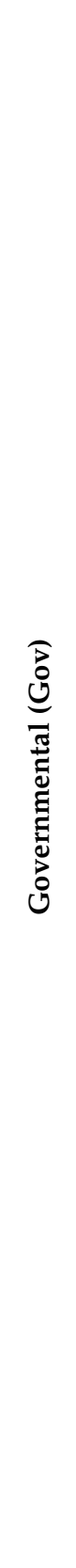 } & $\begin{array}{l}\text { E15. Provide government } \\
\text { sponsored platforms } \\
\text { supporting SMEs }\end{array}$ & $\mathrm{E}$ & $\begin{array}{l}\text { Training, tutoring, consultation, } \\
\text { awareness programs, and } \\
\text { business-related incentives to foster } \\
\text { sustainable manufacturing, attract } \\
\text { customers and business partners. }\end{array}$ & {$[23,56,63,65,77,83,86,89]$} & 8 \\
\hline & $\begin{array}{l}\text { E16. Government provided } \\
\text { information and knowledge on } \\
\text { sustainable manufacturing }\end{array}$ & $\mathrm{E}$ & $\begin{array}{l}\text { Developing condensed knowledge } \\
\text { about the adoption of sustainable } \\
\text { manufacturing practices helps to } \\
\text { overcome low awareness and lack of } \\
\text { technical knowledge (e.g., "how to" } \\
\text { guidance materials, case studies, } \\
\text { webinars, workshops). }\end{array}$ & {$[23,64,69,86,89]$} & 5 \\
\hline & $\begin{array}{l}\text { E17. Providing financial } \\
\text { incentives to foster sustainable } \\
\text { manufacturing practices and } \\
\text { the company's growth }\end{array}$ & $\mathrm{E}$ & $\begin{array}{l}\text { To provide financial incentives, such } \\
\text { as subsidies, fewer taxes on raw } \\
\text { materials, reduced import duties on } \\
\text { environmental technologies. }\end{array}$ & $\begin{array}{l}{[23,37-} \\
39,43,63,65,77,86,89,91]\end{array}$ & 11 \\
\hline & $\begin{array}{l}\text { E18. Providing technical } \\
\text { incentives }\end{array}$ & $\mathrm{E}$ & $\begin{array}{l}\text { Provision of technical development } \\
\text { facilities for SMEs and emphasis on } \\
\text { environmental technology } \\
\text { development. }\end{array}$ & {$[38,63,77,83,89]$} & 5 \\
\hline & $\begin{array}{l}\text { E19. Providing financial } \\
\text { accesses to SMEs }\end{array}$ & $\mathrm{E}$ & $\begin{array}{l}\text { Developing financing channels for } \\
\text { SMEs. }\end{array}$ & {$[23,89]$} & 2 \\
\hline & $\begin{array}{l}\text { E20. Government regulations } \\
\text { fostering sustainability } \\
\text { adoption }\end{array}$ & $\mathrm{E}$ & $\begin{array}{l}\text { Law enforcement is important for } \\
\text { effective implementation of policies } \\
\text { and rules. } \\
\text { Strict regulations could force SMEs to } \\
\text { adopt sustainable manufacturing } \\
\text { measures. }\end{array}$ & {$[37-39,43,63,65,85,86,89,91]$} & 10 \\
\hline & $\begin{array}{l}\text { E21. Legislation appropriately } \\
\text { written toward SMEs }\end{array}$ & $\mathrm{E}$ & $\begin{array}{l}\text { Legislation appropriately written will } \\
\text { improve the understanding of law } \\
\text { and its compliance. }\end{array}$ & {$[37,39,43,65,77,89]$} & 6 \\
\hline & $\begin{array}{l}\text { E22. EU policy supporting } \\
\text { corporate sustainability in SMEs }\end{array}$ & $\mathrm{E}$ & $\begin{array}{l}\text { EU support programmes and other } \\
\text { policies offering directions and access } \\
\text { to funding could improve SMEs } \\
\text { corporate sustainability. }\end{array}$ & {$[67,85]$} & 2 \\
\hline & $\begin{array}{l}\text { E23. Institutionalize rewards } \\
\text { recognizing the adoption of } \\
\text { sustainable manufacturing in } \\
\text { SMEs }\end{array}$ & $\mathrm{E}$ & $\begin{array}{l}\text { Design governmental awards that } \\
\text { recognize SMEs striving to improve } \\
\text { sustainability performance. }\end{array}$ & [65] & 1 \\
\hline \multirow{2}{*}{ 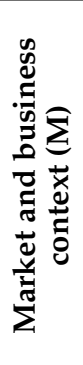 } & $\begin{array}{l}\text { E24. Pressure from market and } \\
\text { clients }\end{array}$ & $\mathrm{E}$ & $\begin{array}{l}\text { Pressure from market enables SMEs } \\
\text { to satisfy customer requirements. } \\
\text { Increasing market demand for } \\
\text { environmentally friendly products. }\end{array}$ & {$[21,63,65,69,83,85,86,89-91]$} & 10 \\
\hline & $\begin{array}{l}\text { E25. Increased support from } \\
\text { large customers }\end{array}$ & $\mathrm{E}$ & $\begin{array}{l}\text { Increased support from OEMs will } \\
\text { require a cultural change in } \\
\text { buyer-supplier relationships. It will } \\
\text { help to improve sustainability } \\
\text { performance and foster SMEs growth. }\end{array}$ & {$[65,89]$} & 2 \\
\hline
\end{tabular}


Table A1. Cont.

Barriers to Sustainable Manufacturing Adoption by Manufacturing SMEs

Cat. Subcategory $\quad$ Origin $\quad$ Description $\quad$ References

Collaboration with industrial associations helps SMEs to stay well

E26. External cooperation E informed and active in their field.

sikewise, SMEs working along
suppliers and customers to improve their sustainability performance.

E27. High energy prices

E

SMEs are likely to reduce energy expenditure if energy prices are high.

$[37,39,43]$

SMEs under strong pressure from

E28. Pressure from supply chains to adopt sustainable supply chains will move toward manufacturing practices E implementing sustainable manufacturing practices and policies are strongly influenced by pressure from supply chains.

E29. Use indicators, monitoring, and controls on energy efficiency

E30. Clarity of information

E31. Technical support from technology suppliers Keeping relevant data makes it
possible to report on progress and
results of energy efficiency practices.

Information about energy efficiency must be adequate to design and implement energy efficiency programs and monitor energy improvement measures.

Technical support from technology suppliers helps to overcome the risks associated with adopting new energy efficiency technologies, such as $[37,39,43]$ production disruptions due to installation.
Industrial associations or similar external stakeholders may increase

E32. Increase the trustworthiness of the information the trustworthiness of information as these are regarded as a trustworthy source by companies.

\begin{tabular}{|c|c|c|c|c|c|}
\hline 玉 & $\begin{array}{l}\text { E33. Reducing manufacturing } \\
\text { costs }\end{array}$ & I & $\begin{array}{l}\text { Cost reduction is vital to guarantee } \\
\text { the continuity of the business. }\end{array}$ & {$[23,37,39,43,64,91]$} & 6 \\
\hline 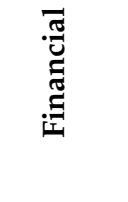 & $\begin{array}{l}\text { E34. Increased awareness of the } \\
\text { economic benefits of sustainable } \\
\text { manufacturing practices }\end{array}$ & I & $\begin{array}{l}\text { Awareness of the economic benefits } \\
\text { of sustainable manufacturing } \\
\text { practices. Implementing sustainable } \\
\text { technology in manufacturing to attain } \\
\text { economic benefits. }\end{array}$ & {$[37,39,43,89]$} & 4 \\
\hline 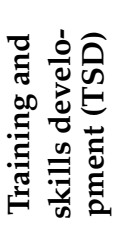 & $\begin{array}{l}\text { E35. Education and training } \\
\text { systems to improve operations }\end{array}$ & I & $\begin{array}{l}\text { Education and training systems are } \\
\text { decisive for improving industry } \\
\text { practices and introducing sustainable } \\
\text { manufacturing concepts and } \\
\text { practices. Sensitization on sustainable } \\
\text { manufacturing practices. }\end{array}$ & {$[37,39,43,64,69,77,83,89]$} & 8 \\
\hline
\end{tabular}




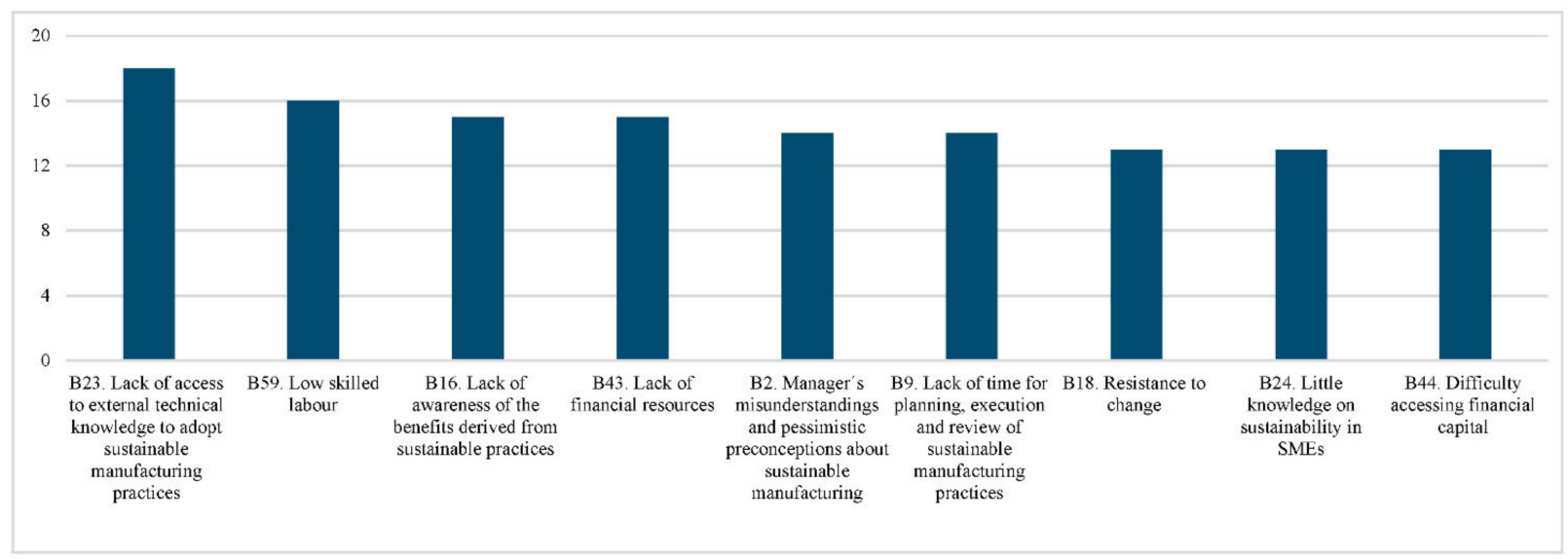

Figure A1. Most frequently occurring barriers in previous literature.

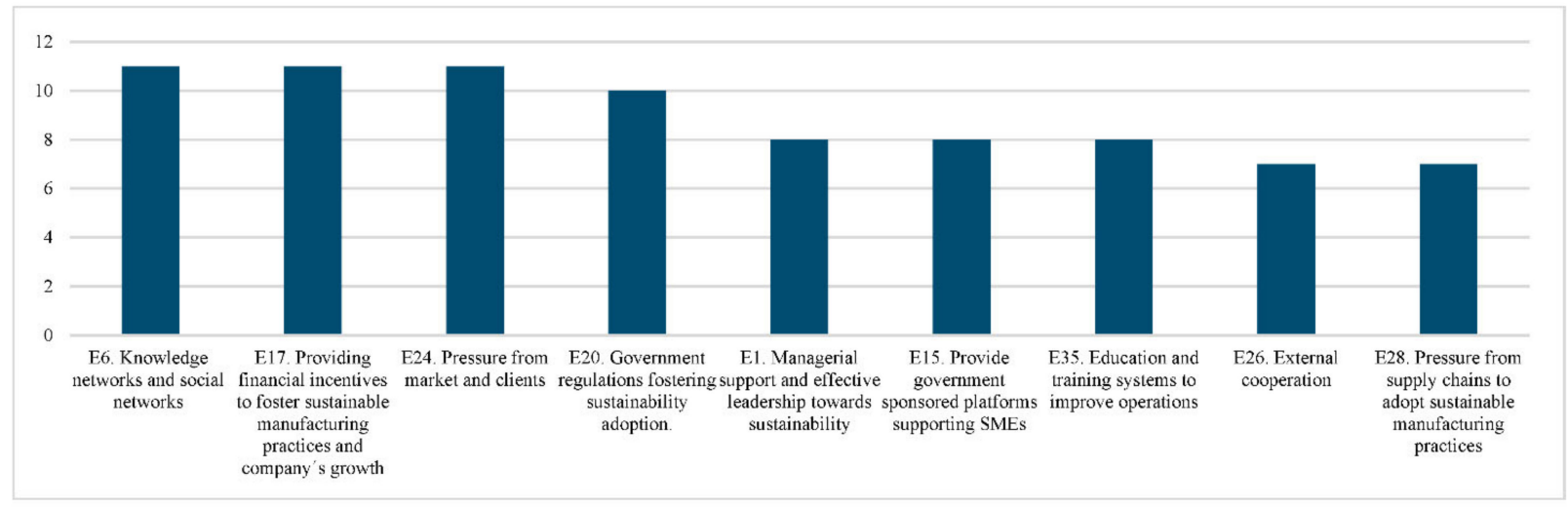

Figure A2. Most frequently occurring enablers in previous literature.

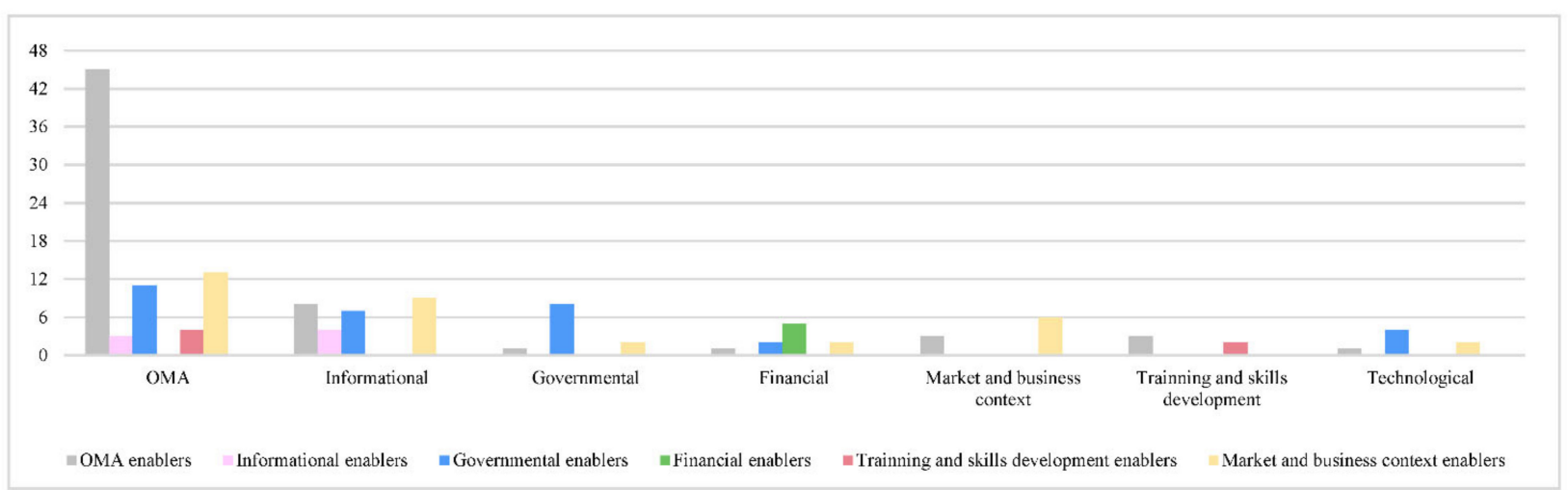

Figure A3. Distribution of enablers expected to mitigate barriers by category.

\section{References}

1. Jansson, J.; Nilsson, J.; Modig, F.; Hed Vall, G. Commitment to Sustainability in Small and Medium-Sized Enterprises: The Influence of Strategic Orientations and Management Values. Bus. Strategy Environ. 2017, 26, 69-83. [CrossRef]

2. Ayyagari, M.; Demirgüç-Kunt, A.; Maksimovic, V. Small vs. Young Firms across the World: Contribution to Employment, Job Creation, and Growth; World Bank: Washington, DC, USA, 2011.

3. Constantinos, C.; Yding Sørensen, S.; Larsen, P.B.; Alexopoulou, S. SMEs and the Environment in the European Union; European Commission: Paris, France, 2010.

4. Hillary, R. Small and Medium-Sized Enterprises and the Environment: Business Imperatives; Greenleaf Publishing: Sheffield, UK, 2000.

5. European Commission. SMEs, Resource Efficiency and Green Markets. Flash Eurobarometer 456; European Commission: Paris, France, 2017. 
6. Revell, A.; Stokes, D.; Chen, H. Small businesses and the environment: Turning over a new leaf? Bus. Strategy Environ. 2010, 19, 273-288. [CrossRef]

7. Brundtland Commission. Report of the World Commission on Environment and Development: Our Common Future; Oxford University Press: Oxford, NY, USA, 1987.

8. De Ron, A.J. Sustainable production: The ultimate result of a continuous improvement. Int. J. Prod. Econ. 1998, 56, 99-110. [CrossRef]

9. Garetti, M.; Taisch, M. Sustainable manufacturing: Trends and research challenges. Prod. Plan. Control 2012, 23, 83-104. [CrossRef]

10. Bhanot, N.; Rao, P.V.; Deshmukh, S.G. Enablers and Barriers of Sustainable Manufacturing: Results from a Survey of Researchers and Industry Professionals. Procedia CIRP 2015, 29, 562-567. [CrossRef]

11. U.S. Department of Commerce. Sustainable Manufacturing Initiatives (SMI): A true Public-Private Dialogue. International Trade Administration. 2010. Available online: https:/ / www.oecd.org/sti/ind/45010349.pdf. (accessed on 15 August 2018).

12. Moldavska, A.; Welo, T. The concept of sustainable manufacturing and its definitions: A content-analysis based literature review. J. Clean. Prod. 2017, 166, 744-755. [CrossRef]

13. Alayón, C.; Säfsten, K.; Johansson, G. Conceptual sustainable production principles in practice: Do they reflect what companies do? J. Clean. Prod. 2017, 141, 693-701. [CrossRef]

14. Welsh, J.A.; White, J.F. A Small Business Is Not a Little Big Business. Harv. Bus. Rev. 1981, 59, $18-26$.

15. Jenkins, H. Small business champions for corporate social responsibility. J. Bus. Ethics 2006, 67, 241-256. [CrossRef]

16. Avlonitis, G.J.; Salavou, H.E. Entrepreneurial orientation of SMEs, product innovativeness, and performance. J. Bus. Res. 2007, 60, 566-575. [CrossRef]

17. Xie, X.M.; Zeng, S.X.; Tam, C.M. Overcoming barriers to innovation in SMEs in China: A perspective based cooperation network. Innov. Manag. Policy Pract. 2010, 12, 298-310. [CrossRef]

18. Terziovski, M. Innovation practice and its performance implications in small and medium enterprises (SMEs) in the manufacturing sector: A resource-based view. Strateg. Manag. J. 2010, 31, 892-902. [CrossRef]

19. Ghazilla, R.; Sakundarini, N.; Abdul-Rashid, S.H.; Ayub, N.S.; Olugu, E.U.; Musa, S.N. Drivers and barriers analysis for green manufacturing practices in Malaysian smes: A preliminary findings. Glob. Conf. Sustain. Manuf. Emerg. Potentials 2015, 26, 658-663. [CrossRef]

20. Sudarsan, R.; Sriram, R.D.; Narayanan, A.; Sarkar, P.; Lee, J.H.; Lyons, K.W.; Kemmerer, S.J. Sustainable Manufacturing: Metrics, Standards, and Infrastructure-Workshop summary. In Proceedings of the 2010 IEEE International Conference on Automation Science and Engineering, Toronto, ON, Canada, 21-14 August 2010; pp. 144-149.

21. Hillary, R. Environmental management systems and the smaller enterprise. J. Clean. Prod. 2004, 12, 561-569. [CrossRef]

22. Shi, H.; Peng, S.Z.; Liu, Y.; Zhong, P. Barriers to the implementation of cleaner production in Chinese SMEs: Government, industry and expert stakeholders' perspectives. J. Clean. Prod. 2008, 16, 842-852. [CrossRef]

23. Henriques, J.; Catarino, J. Motivating towards energy efficiency in small and medium enterprises. J. Clean. Prod. 2016, 139, 42-50. [CrossRef]

24. Oliveira Neto, G.C.; Leite, R.R.; Shibao, F.Y.; Lucato, W.C. Framework to overcome barriers in the implementation of cleaner production in small and medium-sized enterprises: Multiple case studies in Brazil. J. Clean. Prod. 2017, 142, 50-62. [CrossRef]

25. Bhanot, N.; Rao, P.V.; Deshmukh, S.G. An integrated approach for analysing the enablers and barriers of sustainable manufacturing. J. Clean. Prod. 2017, 142, 4412-4439. [CrossRef]

26. Sorrell, S.; Schleich, J.; Scott, S.; O’Malley, E.; Trace, F.; Boede, U. Reducing Barriers to Energy Efficiency in Public and Private Organisation; Electronic Publication, Institute Fraunhofer ISI: Karlsruher, Germany, 2000. Available online: http://publica. fraunhofer.de/documents/N-5674.html (accessed on 15 August 2018).

27. Sorrell, S.; Mallett, A.; Nye, S. Barriers to Industrial Energy Efficiency: A Literature Review; Working Paper no. 10/2011.; United Nations Industrial Development Organization: Vienna, Austria, 2011. Available online: http://sro.sussex.ac.uk/53957/1/WP102 011_Barriers_to_Industrial_Energy_Efficiency_-_A_Literature_Review.pdf. (accessed on 15 August 2018).

28. Cagno, E.; Worrell, E.; Trianni, A.; Pugliese, G. A novel approach for barriers to industrial energy efficiency. Renew. Sustain. Energy Rev. 2013, 19, 290-308. [CrossRef]

29. Roberts, S.J.F.; Ball, P.D. Developing a Library of Sustainable Manufacturing Practices. Procedia CIRP 2014, 15, 159-164. [CrossRef]

30. Natarajan, G.; Wyrick, D. Environmental sustainability in Manufacturing SMEs in West Texas. In Proceedings of the 33rd Annual International Conference of the American Society for Engineering Management 2012, ASEM 2012-Agile Management: Embracing Change and Uncertainty in Engineering Management, Virginia Beach, VA, USA, 17-20 October 2012 ; pp. 856-861.

31. Miras-Rodríguez, M.D.M.; Machuca, J.A.D.; Escobar-Pérez, B. Drivers that encourage environmental practices in manufacturing plants: A comparison of cultural environments. J. Clean. Prod. 2018, 179, 690-703. [CrossRef]

32. Seuring, S.; Müller, M. From a literature review to a conceptual framework for sustainable supply chain management. J. Clean. Prod. 2008, 16, 1699-1710. [CrossRef]

33. Nordin, N.G.A.H.; Hassan, M. Drivers and barriers in sustainable manufacturing implementation in Malaysian manufacturing firms. In Proceedings of the IEEE Conference on Industrial Engineering and Engineering Management, Selangor, Malaysia, 9-12 December 2014; pp. 687-691. 
34. Alayón, C.; Sannö, A.; Säfsten, K.; Johansson, G. Sustainable production in surface treatment SMEs: An explorative study of challenges and enablers from the CEOs perspective. In Proceedings of the Global Cleaner Production and Sustainable Consumption- GCPSC Conference, Sitges, Barcelona, 1-4 November 2015.

35. Walker, H.; Di Sisto, L.; McBain, D. Drivers and barriers to environmental supply chain management practices: Lessons from the public and private sectors. J. Purch. Supply Manag. 2008, 14, 69-85. [CrossRef]

36. Fernández-Viñé, M.B.; Gómez-Navarro, T.; Capuz-Rizo, S.F. Assessment of the public administration tools for the improvement of the eco-efficiency of Small and Medium Sized Enterprises. J. Clean. Prod. 2013, 47, 265-273. [CrossRef]

37. Cagno, E.; Trianni, A.; Spallina, G.; Marchesani, F. Drivers for energy efficiency and their effect on barriers: Empirical evidence from Italian manufacturing enterprises. Energy Effic. 2017, 10, 855-869. [CrossRef]

38. Millar, H.H.; Russell, S.N. The adoption of sustainable manufacturing practices in the Caribbean. Bus. Strategy Environ. 2011, 20, 512-526. [CrossRef]

39. Cagno, E.; Trianni, A.; Abeelen, C.; Worrell, E.; Miggiano, F. Barriers and drivers for energy efficiency: Different perspectives from an exploratory study in the Netherlands. Energy Convers. Manag. 2015, 102, 26-38. [CrossRef]

40. Merriam-Webster. Barrier In Merriam-Webster.com. Available online: https://www.merriam-webster.com/dictionary/barrier (accessed on 15 August 2018).

41. Merriam-Webster. Enabler In Merriam-Webster.com. Available online: https://www.merriam-webster.com/dictionary/enabler (accessed on 15 August 2018).

42. Lee, S.Y.; Klassen, R.D. Drivers and Enablers That Foster Environmental Management Capabilities in Small-and Medium-Sized Suppliers in Supply Chains. Prod. Oper. Manag. 2008, 17, 573-586. [CrossRef]

43. Trianni, A.; Cagno, E.; Marchesani, F.; Spallina, G. Classification of drivers for industrial energy efficiency and their effect on the barriers affecting the investment decision-making process. Energy Effic. 2017, 10, 199-215. [CrossRef]

44. Govindan, K.; Bouzon, M. From a literature review to a multi-perspective framework for reverse logistics barriers and drivers. J. Clean. Prod. 2018, 187, 318-337. [CrossRef]

45. Ardic, O.P.; Mylenko, N.; Saltane, V. Small and Medium Enterprises: A Cross-Country Analysis with a New Data Set; The World Bank: Washington, DC, USA, 2011.

46. OECD. OECD SME and Entrepreneurship Outlook 2005; OECD Publishing: Paris, France, 2005.

47. European Commission. Commission Recommendation of 6 May 2003 Concerning the Definition of Micro, Small and Medium Sized Enterprise; European Commission: Paris, France, 2003; pp. 0036-0041.

48. Ates, A.; Garengo, P.; Cocca, P.; Bititci, U. The development of SME managerial practice for effective performance management. J. Small Bus. Enterp. Dev. 2013, 20, 28-54. [CrossRef]

49. Assarlind, M.; Gremyr, I. Critical factors for quality management initiatives in small-and medium-sized enterprises. Total Qual. Manag. Bus. Excell. 2014, 25, 397-411. [CrossRef]

50. Wielgórka, D. Environmental management in the aspect of sustainable development in micro-, small-, and medium-sized enterprises. Desalination Water Treat. 2016, 57, 982-992. [CrossRef]

51. Jennings, P.; Beaver, G. The performance and competitive advantage of small firms: A management perspective. Int. Small Bus. J. 1997, 15, 63-75. [CrossRef]

52. Cagliano, R.; Spina, G. A comparison of practice-performance models between small manufacturers and subconstractors. Int. J. Oprations Prod. Manag. 2002, 22, 1367-1388. [CrossRef]

53. Jorgensen, A.L.; Knudsen, J.S. Sustainable competitiveness in global value chains: How do small Danish firms behave? Corp. Gov. Int. J. Bus. Soc. 2006, 6, 449-462. [CrossRef]

54. Lozano, R. A Holistic Perspective on Corporate Sustainability Drivers. Corp. Soc. Responsib. Environ. Manag. 2015, 22, 32-44. [CrossRef]

55. Agwa-Ejon, J.; Fore, S. Challenges faced by SMEs in developing countries in implementing cleaner production (CP). Adv. Mater. Res. 2012, 367, 257-263. [CrossRef]

56. Fresner, J.; Morea, F.; Krenn, C.; Aranda Uson, J.; Tomasi, F. Energy efficiency in small and medium enterprises: Lessons learned from 280 energy audits across Europe. J. Clean. Prod. 2017, 142, 1650-1660. [CrossRef]

57. Denyer, D.; Tranfield, D. Chapter 39: Producing a systematic review. In The Sage Handbook of Organizational Research Methods; Buchanan, D., Bryman, A.E., Eds.; Sage Publications Ltd.: Thousand Oaks, CA, USA, 2009; pp. 671-689.

58. Jesson, J.K.; Matheson, L.; Lacey, F.M. Doing Your Literature Review. Traditional and Systematic Techniques; Sage: London, UK, 2011.

59. Thomé, A.M.T.; Scavarda, L.F.; Scavarda, A.J. Conducting systematic literature review in operations management. Prod. Plan. Control 2016, 27, 408-420. [CrossRef]

60. Hsieh, H.F.; Shannon, S.E. Three Approaches to Qualitative Content Analysis. Qual. Health Res. 2005, 15, 1277-1288. [CrossRef] [PubMed]

61. Miles, M.B.; Huberman, A.M.; Saldaña, J. Qualitative Data Analysis: An Expanded Sourcebook; SAGE Publications: Thousand Oaks, CA, SUA, 2019.

62. Seuring, S.; Gold, S. Conducting content-Analysis based literature reviews in supply chain management. Supply Chain Manag. Int. J. 2012, 17, 544-555. [CrossRef]

63. Coppa, M.; Sriramesh, K. Corporate social responsibility among SMEs in Italy. Public Relat. Rev. 2013, 39, 30-39. [CrossRef] 
64. Thomas, A.; Francis, M.; John, E.; Davies, A. Identifying the characteristics for achieving sustainable manufacturing companies. J. Manuf. Technol. Manag. 2012, 23, 426-440. [CrossRef]

65. Agan, Y.; Acar, M.F.; Borodin, A. Drivers of environmental processes and their impact on performance: A study of Turkish SMEs. J. Clean. Prod. 2013, 51, 23-33. [CrossRef]

66. Ashton, W.S.; Panero, M.A.; Izquierdo Cruz, C.; Hurtado Martin, M. Financing resource efficiency and cleaner production in Central America. Clean. Technol. Env. Policy 2018, 20, 53-63. [CrossRef]

67. Tomšič, N.; Bojnec, Š.; Simčič, B. Corporate sustainability and economic performance in small and medium sized enterprises. J. Clean. Prod. 2015, 108, 603-612. [CrossRef]

68. Williamson, D.; Lynch-Wood, G.; Ramsay, J. Drivers of Environmental Behaviour in Manufacturing SMEs and the Implications for CSR. J. Bus. Ethics 2006, 67, 317-330. [CrossRef]

69. Triguero, A.; Moreno-Mondéjar, L.; Davia, M.A. The influence of energy prices on adoption of clean technologies and recycling: Evidence from European SMEs. Energy Econ. 2014, 46, 246-257. [CrossRef]

70. De Barros, R.L.P.; De Paiva, M.D.F.F.; Sisinno, C.L.S. Cleaner production challenges in Brazilian SMEs. Ind. Environ. 2003, 26, 26-28.

71. Jabbour, C.J.C.; Puppim-De-Oliveira, J.A. Barriers to environmental management in clusters of small businesses in Brazil and Japan: From a lack of knowledge to a decline in traditional knowledge. Int. J. Sustain. Dev. World Ecol. 2012, 19, $247-257$. [CrossRef]

72. Aloulou, W.; Fayolle, A. A Conceptual Approach of the Entrepreneurial Orientation Within the Small Business Context. J. Enterprising Cult. 2005, 13, 21-45. [CrossRef]

73. Cagliano, R.; Blackmon, K.; Voss, C. Small firms under microscope: International differences in production/operations management practices and performance. Integr. Manuf. Syst. 2001, 12, 469-482. [CrossRef]

74. Huang, A.; Badurdeen, F. Sustainable Manufacturing Performance Evaluation: Integrating Product and Process Metrics for Systems Level Assessment. Procedia Manuf. 2017, 8, 563-570. [CrossRef]

75. Mittal, V.K.; Sangwan, K.S. Prioritizing Barriers to Green Manufacturing: Environmental, Social and Economic Perspectives. Procedia CIRP 2014, 47, 559-564. [CrossRef]

76. Mittal, V.K.; Sangwan, K.S.; Herrmann, C. Comparison of Drivers and Barriers to Green Manufacturing: A Case of India and Germany. In 20th CIRP International Conference on Life Cycle Engineering; Springer: Sinagpore, 2013; pp. 723-728. [CrossRef]

77. Ramjeawon, T. A case study of cleaner production opportunities in small and medium enterprises on the island of Mauritius. Electron. Green J. 2004, 1, 20. [CrossRef]

78. Spence, L.J.; Schmidpeter, R. SMEs, Social Capital and the Common Good. J. Bus. Ethics 2003, 45, 93-108. [CrossRef]

79. Shah, S.F.H.; Nazir, T.; Zaman, K.; Shabir, M. Factors affecting the growth of enterprises: A survey of the literature from the perspective of small- and medium-sized enterprises. J. Enterp. Transform. 2013, 3, 53-75. [CrossRef]

80. Murillo-Luna, J.L.; Garcés-Ayerbe, C.; Rivera-Torres, P. Barriers to the adoption of proactive environmental strategies. J. Clean. Prod. 2011, 19, 1417-1425. [CrossRef]

81. Balzarova, M.A.; Castka, P. Underlying mechanisms in the maintenance of ISO 14001 environmental management system. J. Clean. Prod. 2008, 16, 1949-1957. [CrossRef]

82. Trianni, A.; Cagno, E.; Worrell, E.; Pugliese, G. Empirical investigation of energy efficiency barriers in Italian manufacturing SMEs. Energy 2013, 49, 444-458. [CrossRef]

83. Seidel, M.; Seidel, R.; Tedford, D.; Cross, R.; Wait, L.; Hämmerle, E. Overcoming barriers to implementing environmentally benign manufacturing practices: Strategic tools for SMEs. Environ. Qual. Manag. 2009, 18, 37-55. [CrossRef]

84. Hasan, M.N. Measuring and understanding the engagement of Bangladeshi SMEs with sustainable and socially responsible business practices: An ISO 26000 perspective. Soc. Responsib. J. 2016, 12, 584-610. [CrossRef]

85. Seidel-Sterzik, H.; McLaren, S.; Garnevska, E. Effective life cycle management in SMEs: Use of a sector-based approach to overcome barriers. Sustainability 2018, 10, 359. [CrossRef]

86. Gombault, M.; Versteege, S. Cleaner production in SMEs through a partnership with (local) authorities: Successes from the Netherlands. J. Clean. Prod. 1999, 7, 249-261. [CrossRef]

87. Hitchens, D.; Clausen, J.; Trainor, M.; Keil, M.; Thankappan, S. Competitiveness, environmental performance and management of SMEs. Greener Manag. Int. 2003, 44, 45-57. [CrossRef]

88. Hsu, J.L.; Cheng, M.C. What Prompts Small and Medium Enterprises to Engage in Corporate Social Responsibility? A Study from Taiwan. Corp. Soc. Responsib. Environ. Manag. 2012, 19, 288-305. [CrossRef]

89. Frijns, J.; Van Vliet, B. Small-scale industry and cleaner production strategies. World Dev. 1999, 27, 967-983. [CrossRef]

90. Aboelmaged, M. The drivers of sustainable manufacturing practices in Egyptian SMEs and their impact on competitive capabilities: A PLS-SEM model. J. Clean. Prod. 2018, 175, 207-221. [CrossRef]

91. Gandhi, N.S.; Thanki, S.J.; Thakkar, J.J. Ranking of drivers for integrated lean-green manufacturing for Indian manufacturing SMEs. J. Clean. Prod. 2018, 171, 675-689. [CrossRef] 